\title{
Different Coordination Modes of an Aryl-substituted Hydrotris(pyrazolyl)borate Ligand in Rhodium and Iridium Complexes
}

Salvador Conejero, Ana Cristina Esqueda, José E. V. Valpuesta, Eleuterio Álvarez, Celia Maya, Ernesto Carmona*

Instituto de Investigaciones Químicas, Departamento de Química Inorgánica, CSIC and Universidad de Sevilla, Avda. Américo Vespucio 49, 41092 Sevilla, Spain

Keywords: Rhodium-Iridium-tris(pyrazolyl)borate-C-H activation

"This paper is dedicated to Professor Robert G. Bergman, in recognition of his outstanding contributions to Organometallic Chemistry”

\begin{abstract}
Complexes $\mathrm{Tp}^{\text {tol }} \mathrm{Rh}\left(\mathrm{C}_{2} \mathrm{H}_{4}\right)_{2}$ (1a) and $\mathrm{Tp}^{\text {tol }} \mathrm{Rh}\left(\mathrm{CH}_{2}=\mathrm{C}(\mathrm{Me}) \mathrm{C}(\mathrm{Me})=\mathrm{CH}_{2}\right)$ (1) b have been prepared by reaction of $\mathrm{KTp}^{\text {tol }}$ with the appropriate $\left[\mathrm{RhCl}(\text { olefin })_{2}\right]_{2}$ dimer $\left(\mathrm{Tp}^{\text {tol }}\right.$ means hydrotris(3-p-tolylpyrazol-1-yl)borate). The two complexes show a dynamic behaviour that involves exchange between $\kappa^{2}$ and $\kappa^{3}$ coordination modes of the $\mathrm{Tp}^{\text {tol }}$ ligand. The iridium analogue, $\operatorname{Tp}^{\text {tol }} \operatorname{Ir}\left(\mathrm{CH}_{2}=\mathrm{C}(\mathrm{Me}) \mathrm{CH}=\mathrm{CH}_{2}\right)$ (2) has also been synthesized, and has been converted into the $\operatorname{Ir}(\mathrm{III})$ dinitrogen complex $\left[\left(\kappa^{4}-N, N^{\prime}, N^{\prime}, C-\mathrm{Tp}{ }^{\mathrm{tol}}\right) \operatorname{Ir}(\mathrm{Ph})\left(\mathrm{N}_{2}\right)(3)\right.$ by irradiation with UV light under a dinitrogen atmosphere. Compound $\mathbf{3}$ constitutes a rare example of $\mathrm{Ir}(\mathrm{III})-\mathrm{N}_{2}$ complex structurally characterized by X-ray crystallography. Its $\mathrm{N}_{2}$ ligand can be easily substituted by acetonitrile or ethylene upon heating and denticity changes in the $\mathrm{Tp}^{\text {tol }}$ ligand, from $\kappa^{4}-N, N^{\prime}, N^{\prime}, C$ (monometallated $\mathrm{Tp}^{\text {tol }}$, from now on represented as $\mathrm{Tp}^{\text {tol' }}$ ) to $\kappa^{5}-N, N^{\prime}, N^{\prime \prime}, C, C$ ' (dimetallated $\mathrm{Tp}^{\text {tol }}$ ligand, represented as $\mathrm{Tp}^{\text {tol") }}$ ) have been observed. When complex 3 is heated in the presence of acetylene, dimerization of the alkyne takes place to yield the enyne complex $\left[\left(\kappa^{5}-N, N^{\prime}, N^{\prime}, C, C\right.\right.$ '-

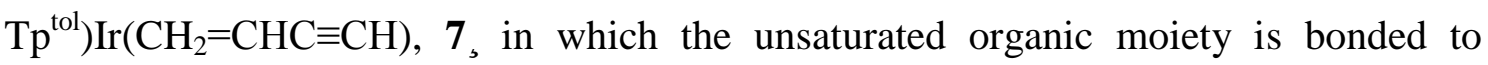
iridium through the carbon-carbon double bond.
\end{abstract}




\section{Introduction}

Hydrotris(pyrazolyl)borate ligands are among the most widely used anionic ligands in different areas of coordination and organometallic chemistry [1]. Since they were first reported by Trofimenko more than 40 years ago [2], the number of structurally modified hydrotris(pyrazolyl)borates that have been synthesized is enormous [3]. However, very often either the unsubstituted Tp (Tp stands for the parent hydrotris(pyrazolyl)borate), or $\mathrm{Tp}^{\mathrm{Me} 2}\left(\mathrm{Tp}^{\mathrm{Me} 2} \equiv\right.$ hydrotris(3,5-dimethylpyrazolyl)borate) have been the ligands of choice when searching for new metal complexes and reactivity modes. These two ligands usually coordinate to metal centres in a $\kappa^{2}$ or $\kappa^{3}$ fashion, although " $\kappa^{0}$ " and $\kappa^{1}$ coordination modes have been also reported [4]. Less attention has been paid to other 3substituted Tp' ligands that can undergo metallation to act as $\kappa^{4}$ or $\kappa^{5}$ ligands [5].

We have previously reported that $\mathrm{Tp}^{\mathrm{Ph}}$ (hydrotris(3-phenylpyrazolyl)borate) may adopt unusual $\mathrm{k}^{5}$-coordination through double metallation of two phenyl rings at the pyrazolyl fragments [5i,j]. More recently, we have observed a similar behaviour for the $\mathrm{Tp}^{\text {tol }}$ system $\left(\mathrm{Tp}^{\text {tol }} \equiv\right.$ hydrotris( $p$-tolylpyrazolyl)borate), which under certain reaction conditions experiences mono or double metallation of the tolyl groups [6]. Some of the complexes that these metallated Tp' ligands form with group 9 transition metals are particularly active in $\mathrm{C}-\mathrm{H}$ bond activation processes [7]. For instance, in recent years, we have shown that metallated Tp' moieties may participate in the tautomerization of pyridines to N-heterocyclic carbenes [8]. Moreover, different reactivity patterns have been disclosed when $\mathrm{Tp}^{\mathrm{Me} 2} \mathrm{Ir}$ fragments are compared with related iridium complexes containing aryl substituted $\mathrm{Tp}$ ligands such $\mathrm{Tp}^{\mathrm{Ms}} \quad\left(\mathrm{Tp}^{\mathrm{Ms}} \equiv \operatorname{hydrotris}(3-\right.$ mesitylpyrazolyl)borate) and $\mathrm{Tp}^{\mathrm{Ph}}$. As a way of an example, a $\mathrm{Tp}^{\mathrm{Ph}} \mathrm{Ir}$ fragment promotes double $\mathrm{C}-\mathrm{H}$ activation of diethyl ether to yield the corresponding Fischer-type carbene $[\mathrm{Ir}]=\mathrm{C}\left(\mathrm{CH}_{3}\right)(\mathrm{OEt})$, while a related $\mathrm{Tp}^{\mathrm{Me} 2}$ Ir unit gives a complex reaction mixture when heated in the presence of diethyl ether $[7 \mathrm{i}, \mathrm{j}]$. Another interesting case that exemplifies the effects of substituents at the Tp ligand is the already mentioned tautomerization of pyridines into N-heterocyclic carbenes promoted by Tp'Ir complexes. Thus, whereas a doubly metallated $\mathrm{Tp}^{\mathrm{Ms}} \mathrm{Ir}$ fragment promotes the conversion of pyridine into an $\mathrm{N}$ heterocyclic carbene (in competition with formation of isomeric $\mathrm{N}$-adduct), the related $\mathrm{Tp}^{\mathrm{Me} 2} \mathrm{Ir}$ derivatives yield exclusively $\mathrm{N}$-adducts $[8,9]$. 
As a natural extension of this work we have investigated the coordination properties of $\mathrm{Tp}^{\text {tol }}$ ligands towards rhodium and iridium. We have also explored the reactivity of the iridium counterparts, and we report herein the synthesis of a dinitrogen complex stabilized by a monometallated $\mathrm{Tp}^{\text {tol' }}$ ligand, including its X-ray structure and reactivity $\left(\mathrm{Tp}^{\mathrm{tol}}\right.$ ' $s$ tands for $\mathrm{Tp}^{\mathrm{tol}}$ metallated in an ortho position of a $p$-tolyl substituent).

\section{Experimental}

\subsection{General considerations.}

All syntheses were performed under an argon atmosphere using standard Schlenk techniques, employing dry solvents and glassware. Spectra were referenced to external $\mathrm{SiMe}_{4}(\delta 0 \mathrm{ppm})$ using the residual protio solvent peaks as internal standards $\left({ }^{1} \mathrm{H} \mathrm{NMR}\right.$ experiments) or the characteristic resonances of the solvent nuclei $\left({ }^{13} \mathrm{C} \mathrm{NMR}\right.$ experiments). ${ }^{11} \mathrm{~B}\left\{{ }^{1} \mathrm{H}\right\}$ NMR has been referenced to $\mathrm{BF}_{3} \cdot \mathrm{OEt}_{2}$. Spectral assignments were made by routine one- and two-dimensional NMR experiments where appropriate. The crystal structures were determined in a Bruker-AXSX8Kappa diffractometer. Compounds $\left[\mathrm{RhCl}\left(\mathrm{C}_{2} \mathrm{H}_{4}\right)_{2}\right]_{2}[10],\left[\operatorname{IrCl}(\mathrm{coe})_{2}\right]_{2}$ [11], and $\mathrm{KTp}^{\text {tol }}$ [12] were prepared as previously described.

\subsection{Synthesis of $\mathrm{Tp}^{\text {tol }} \mathrm{Rh}\left(\mathrm{CH}_{2}=\mathrm{CH}_{2}\right)_{2}(\mathrm{la})$.}

$\mathrm{KTp}^{\text {tol }}(184 \mathrm{mg}, 0.38 \mathrm{mmol})$ is added to a solution of $\left[\mathrm{RhCl}\left(\mathrm{C}_{2} \mathrm{H}_{4}\right)_{2}\right]_{2}(75 \mathrm{mg}, 0.19$ $\mathrm{mmol})$ in THF $(20 \mathrm{~mL})$. After stirring for a period of $3 \mathrm{~h}$ the solvent is removed under vacuum and complex 1a is extracted with a 2:1 mixture of $\mathrm{CH}_{2} \mathrm{Cl}_{2}-\mathrm{Et}_{2} \mathrm{O}$. The solution is filtered through Celite and the solvent is then evaporated under reduced pressure to obtain an orange solid (Yield, 92\%).

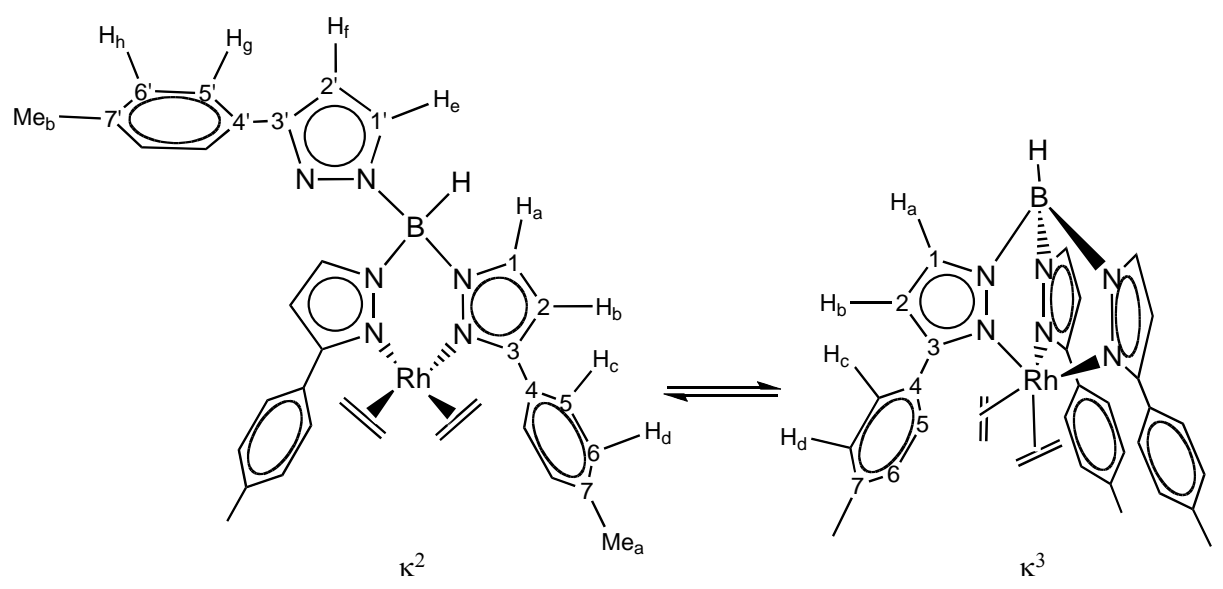


$\kappa^{2}-T p^{\text {tol }}$ :

IR (Nujol): $v(\mathrm{~B}-\mathrm{H}) 2390 \mathrm{~cm}^{-1} .{ }^{11} \mathbf{B}\left\{{ }^{1} \mathbf{H}\right\}$ NMR $\left(96 \mathrm{MHz}, \mathrm{C}_{6} \mathrm{D}_{6}, 25{ }^{\circ} \mathrm{C}\right) \delta:-1.9 .{ }^{1} \mathbf{H}$ NMR $\left(400 \mathrm{MHz}, \mathrm{CD}_{2} \mathrm{Cl}_{2}, 25^{\circ} \mathrm{C}\right) \delta: 8.15\left(\mathrm{~d}, 1 \mathrm{H}, \mathrm{H}_{\mathrm{e}}\right), 8.07\left(\mathrm{~d}, 4 \mathrm{H}, \mathrm{H}_{\mathrm{c}}\right), 7.88\left(\mathrm{~d}, 2 \mathrm{H}, \mathrm{H}_{\mathrm{a}}\right), 7.48$ (d, $4 \mathrm{H}, \mathrm{H}_{\mathrm{d}}$ ), 7.47 (d, $2 \mathrm{H}, \mathrm{H}_{\mathrm{g}}$ ), $7.26\left(\mathrm{~d}, 2 \mathrm{H}, \mathrm{H}_{\mathrm{h}}\right), 6.79$ (d, $\left.1 \mathrm{H}, \mathrm{H}_{\mathrm{f}}\right), 6.35\left(\mathrm{~d}, 2 \mathrm{H}, \mathrm{H}_{\mathrm{b}}\right.$ ), 4.80 (br, $1 \mathrm{H}, \mathrm{BH}$ ), 2.70 (m br, $8 \mathrm{H}$, ethylene), 2.55 (s, $6 \mathrm{H}, 2 \mathrm{Me}_{\mathrm{a}}$ ), 2.42 (s, $3 \mathrm{H}, \mathrm{Me}_{\mathrm{b}}$ ). ${ }^{13} \mathbf{C}\left\{{ }^{1} \mathbf{H}\right\}$ NMR $\left(100 \mathrm{MHz}, \mathrm{CD}_{2} \mathrm{Cl}_{2}, 25^{\circ} \mathrm{C}\right) \delta: 154.3\left(\mathrm{C}^{3}\right), 153.9\left(\mathrm{C}^{3}\right), 139.3\left(\mathrm{CH}^{1}\right)$, $139.1\left(\mathrm{C}^{7}\right), 137.4\left(\mathrm{C}^{7}\right), 136.0\left(\mathrm{CH}^{5}\right), 132.4\left(\mathrm{C}^{4}\right), 130.5\left(\mathrm{C}^{4}\right), 129.5\left(\mathrm{CH}^{6}\right), 129.4$ $\left(\mathrm{CH}^{6}\right), 128.8\left(\mathrm{CH}^{5}\right), 126.0\left(\mathrm{CH}^{1}\right), 105.3\left(\mathrm{CH}^{2}\right), 102.3\left(\mathrm{CH}^{2}\right), 65.4\left(\mathrm{~d},{ }^{1} J_{\mathrm{CRh}}=12 \mathrm{~Hz}\right.$, $\left.=\mathrm{CH}_{2}\right), 21.5\left(\mathrm{Me}_{\mathrm{a}}\right), 21.3\left(\mathrm{Me}_{\mathrm{b}}\right)$. Anal. calcd. for $\mathrm{C}_{36} \mathrm{H}_{38} \mathrm{BN}_{6} \mathrm{Rh}: \mathrm{C}, 64.7 ; \mathrm{H}, 5.7 ; \mathrm{N}, 12.6$. Found: C, 65.7; H, 5.4; N, 12.5 .

$\kappa^{3}-T p^{\text {tol }}$ :

IR (Nujol): $v$ (B-H) $2420 \mathrm{~cm}^{-1} .{ }^{1} \mathbf{H}$ NMR $\left(400 \mathrm{MHz}, \mathrm{CD}_{2} \mathrm{Cl}_{2}, 25^{\circ} \mathrm{C}\right) \delta: 7.99(\mathrm{~d}, 6 \mathrm{H}$, $\mathrm{H}_{\mathrm{d}}$ ), $7.90\left(\mathrm{~d}, 3 \mathrm{H}, \mathrm{H}_{\mathrm{a}}\right.$ ), 7.37 (d, $6 \mathrm{H}, \mathrm{H}_{\mathrm{c}}$ ), 6.57 (d, $3 \mathrm{H}, \mathrm{H}_{\mathrm{b}}$ ), 2.47 (s, $\left.9 \mathrm{H}, 3 \mathrm{Me}\right), 2.78$ (br, $\left.\left.8 \mathrm{H},=\mathrm{CH}_{2}\right) .{ }^{13} \mathrm{C}^{1}{ }^{1} \mathrm{H}\right\}$ NMR $\left(100 \mathrm{MHz}, \mathrm{CD}_{2} \mathrm{Cl}_{2}, 25^{\circ} \mathrm{C}\right) \delta: 154.5\left(\mathrm{C}^{3}\right), 138.4\left(\mathrm{C}^{7}\right), 137.7$ $\left(\mathrm{CH}^{1}\right), 131.4\left(\mathrm{C}^{4}\right), 129.5\left(\mathrm{CH}^{6}\right), 127.8\left(\mathrm{CH}^{5}\right), 104.8\left(\mathrm{CH}^{2}\right), 66.4\left(\mathrm{~d},{ }^{1} J_{\mathrm{CRh}}=12 \mathrm{~Hz}\right.$, $\left.=\mathrm{CH}_{2}\right), 21.4(\mathrm{Me})$.

\subsection{Synthesis of $\mathrm{Tp}^{\mathrm{tol}} \mathrm{Rh}\left(\mathrm{CH}_{2}=\mathrm{C}(\mathrm{Me}) \mathrm{C}(\mathrm{Me})=\mathrm{CH}_{2}\right)(\mathbf{l} \boldsymbol{b})$.}

2,3-dimethylbutadiene (200 $\mu \mathrm{l})$ is added, at $0{ }^{\circ} \mathrm{C}$, to a solution of $\left[\mathrm{RhCl}\left(\mathrm{C}_{2} \mathrm{H}_{4}\right)_{2}\right]_{2}(75$ $\mathrm{mg}, 0.19 \mathrm{mmol})$ in $\mathrm{THF}(20 \mathrm{~mL})$. The mixture is stirred at this temperature for $15 \mathrm{~min}$ and for a further period of $1.5 \mathrm{~h}$ at $20^{\circ} \mathrm{C}$. After this time, a solution of $\mathrm{KTp}^{\text {tol }}(184 \mathrm{mg}$, $0.38 \mathrm{mmol})$ in THF $(20 \mathrm{~mL})$ is added and the mixture stirred for a period of $3 \mathrm{~h}$. The solvent is removed under vacuum and complex $\mathbf{1 b}$ is extracted with a 2:1 mixture of $\mathrm{CH}_{2} \mathrm{Cl}_{2}-\mathrm{Et}_{2} \mathrm{O}$. The solution is filtered through Celite and the solvent evaporated under reduced pressure to obtain an orange solid (Yield, 92\%). 

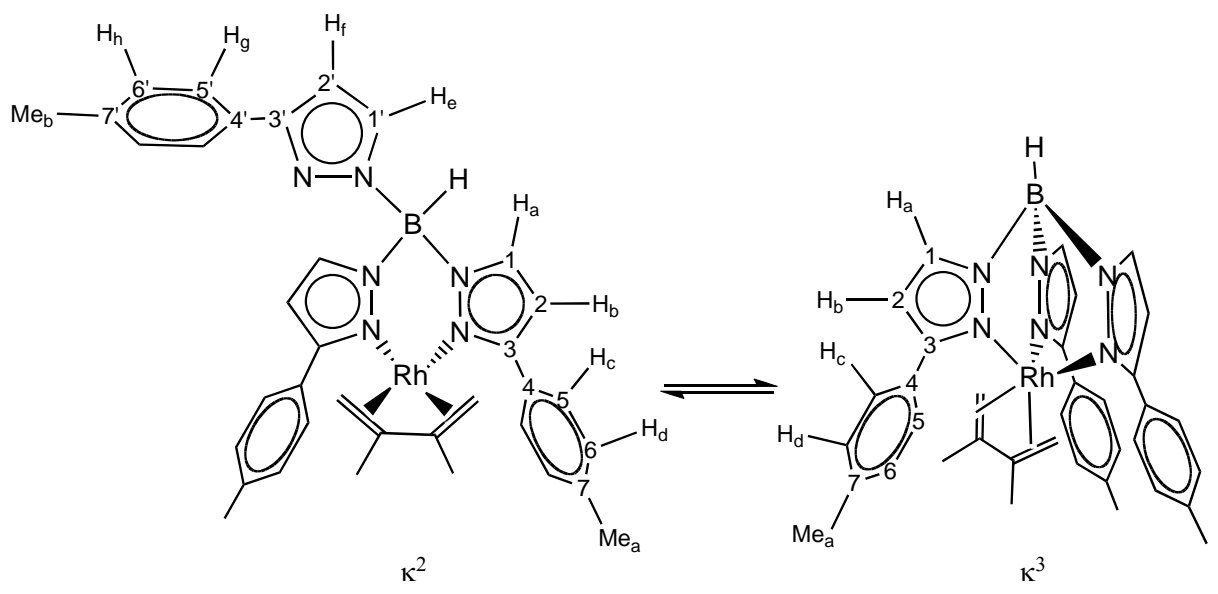

$\kappa^{2}-T p^{\text {tol }}$

IR (Nujol): $v(\mathrm{~B}-\mathrm{H}) 2395 \mathrm{~cm}^{-1} .{ }^{11} \mathbf{B}\left\{{ }^{1} \mathbf{H}\right\}$ NMR $\left(96 \mathrm{MHz}, \mathrm{C}_{6} \mathrm{D}_{6}, 25{ }^{\circ} \mathrm{C}\right) \delta:-1.9 .{ }^{1} \mathbf{H}$ NMR $\left(400 \mathrm{MHz}, \mathrm{CD}_{2} \mathrm{Cl}_{2}, 25^{\circ} \mathrm{C}\right) \delta: 8.13\left(\mathrm{~d}, 4 \mathrm{H}, \mathrm{H}_{\mathrm{c}}\right), 7.96\left(\mathrm{~d}, 1 \mathrm{H}, \mathrm{H}_{\mathrm{e}}\right), 7.85\left(\mathrm{~d}, 2 \mathrm{H}, \mathrm{H}_{\mathrm{g}}\right), 7.42$ (d, $\left.4 \mathrm{H}, \mathrm{H}_{\mathrm{d}}\right), 7.26\left(\mathrm{~d}, 2 \mathrm{H}, \mathrm{H}_{\mathrm{a}}\right), 7.24\left(\mathrm{~d}, 2 \mathrm{H}, \mathrm{H}_{\mathrm{h}}\right), 6.74\left(\mathrm{~d}, 1 \mathrm{H}, \mathrm{H}_{\mathrm{f}}\right), 6.45\left(\mathrm{~d}, 2 \mathrm{H}, \mathrm{H}_{\mathrm{b}}\right)$, 4.80 (br, $1 \mathrm{H}, \mathrm{BH}$ ), 2.51 (s, $6 \mathrm{H}, 2 \mathrm{Me}_{\mathrm{a}}$ ), 2.41 (s, $3 \mathrm{H}, \mathrm{Me}_{\mathrm{b}}$ ), 2.51 (s, $6 \mathrm{H}, 2=\mathrm{CMe}$ ), 1.65, 0.57 (s, $2 \mathrm{H}$ each, $\left.=\mathrm{CH}_{2}\right) \cdot{ }^{13} \mathbf{C}\left\{{ }^{1} \mathbf{H}\right\}$ NMR $\left(100 \mathrm{MHz}, \mathrm{CD}_{2} \mathrm{Cl}_{2}, 2{ }^{\circ} \mathrm{C}\right) \delta: 154.9\left(\mathrm{C}^{3}\right)$, 154.2 $\left(\mathrm{C}^{3{ }^{\prime}}\right), 139.0\left(\mathrm{CH}^{1}\right), 138.8\left(\mathrm{C}^{7}\right), 137.4\left(\mathrm{C}^{7}\right), 135.5\left(\mathrm{CH}^{1}\right), 137.3\left(\mathrm{C}^{4^{\prime}}\right), 132.0\left(\mathrm{C}^{4}\right)$, $129.5\left(\mathrm{CH}^{6}\right), 129.0\left(\mathrm{CH}^{6}\right), 128.8\left(\mathrm{CH}^{5}\right), 125.9\left(\mathrm{CH}^{5}\right), 104.1\left(\mathrm{CH}^{2}\right), 102.1\left(\mathrm{CH}^{2}\right), 98.4$ $\left(\mathrm{d},{ }^{1} J_{\mathrm{CRh}}=10 \mathrm{~Hz}, C=\mathrm{CH}_{2}\right), 47.6\left(\mathrm{~d}^{1} J_{\mathrm{CRh}}=13 \mathrm{~Hz},=C \mathrm{Me}\right), 21.5\left(\mathrm{Me}_{\mathrm{a}}\right), 21.3\left(\mathrm{Me}_{\mathrm{b}}\right), 19.5$ (=CMe). Anal. calcd. for $\mathrm{C}_{34} \mathrm{H}_{36} \mathrm{BN}_{6} \mathrm{Rh}_{\mathrm{Et}} \mathrm{O}: \mathrm{C}, 63.7 ; \mathrm{H}, 6.5 ; \mathrm{N}$, 11.7. Found: C, 64.4; $\mathrm{H}, 6.4 ; \mathrm{N}, 11.9$.

$\kappa^{3}-T p^{\text {tol: }}$

IR (Nujol): $v(\mathrm{~B}-\mathrm{H}) 2415 \mathrm{~cm}^{-1} .{ }^{1} \mathbf{H}$ NMR $\left(400 \mathrm{MHz}, \mathrm{CD}_{2} \mathrm{Cl}_{2}, 25^{\circ} \mathrm{C}\right) \delta: 7.93(\mathrm{~d}, 6 \mathrm{H}$, $\mathrm{H}_{\mathrm{c}}$ ), $7.62\left(\mathrm{~d}, 3 \mathrm{H}, \mathrm{H}_{\mathrm{a}}\right), 7.33\left(\mathrm{~d}, 6 \mathrm{H}, \mathrm{H}_{\mathrm{d}}\right), 6.58\left(\mathrm{~d}, 3 \mathrm{H}, \mathrm{H}_{\mathrm{b}}\right.$ ), 4.80 (br, $\left.1 \mathrm{H}, \mathrm{BH}\right), 2.47$ (s, 9 H, $3 \mathrm{Me}_{\mathrm{a}}$ ), 1.50, 0.32 (s, $2 \mathrm{H}$ each, $\mathrm{CH}_{2}$ ), 1.12 (s, $6 \mathrm{H}, 2 \mathrm{Me}_{\mathrm{b}}$ ). ${ }^{13} \mathbf{C}\left\{{ }^{1} \mathbf{H}\right\}$ NMR (100 $\left.\mathrm{MHz}, \mathrm{CD}_{2} \mathrm{Cl}_{2}, 25^{\circ} \mathrm{C}\right) \delta: 155.2\left(\mathrm{C}^{3}\right), 138.2\left(\mathrm{C}^{7}\right), 137.3\left(\mathrm{CH}^{1}\right), 132.6\left(\mathrm{C}^{4}\right), 129.1\left(\mathrm{CH}^{6}\right)$, $128.1\left(\mathrm{CH}^{5}\right), 104.4\left(\mathrm{CH}^{2}\right), 98.0\left(\mathrm{~d},{ }^{1} J_{\mathrm{CRh}}=9 \mathrm{~Hz},=C \mathrm{Me}\right), 47.0\left(\mathrm{~d},{ }^{1} J_{\mathrm{CRh}}=12 \mathrm{~Hz},=\mathrm{CH}_{2}\right)$, $21.4\left(\mathrm{Me}_{\mathrm{a}}\right), 18.5(=\mathrm{CMe})$.

2.4 Synthesis of complex $\operatorname{Tp}^{\text {tol }} \mathrm{Ir}\left(\mathrm{CH}_{2}=\mathrm{C}(\mathrm{Me}) \mathrm{CH}=\mathrm{CH}_{2}\right)$ (2).

Isoprene $(4 \mathrm{~mL})$ is added at $20{ }^{\circ} \mathrm{C}$ to a suspension of $\left[\mathrm{IrCl}(\mathrm{coe})_{2}\right]_{2}(0.92 \mathrm{~g}, 1.03 \mathrm{mmol})$ in $\mathrm{CH}_{2} \mathrm{Cl}_{2}(80 \mathrm{~mL})$. The resulting mixture is stirred for $30 \mathrm{~min}$ and after this time $\mathrm{KTp}^{\text {tol }}$ $(1.08 \mathrm{~g}, 2.06 \mathrm{mmol})$ is added. After stirring for a period of $24 \mathrm{~h}$ the solution is 
centrifuged and the supernatant separated and evaporated to dryness. The solid residue is washed with hexane ( $3 \times 20 \mathrm{~mL})$ and dried under vacuum to obtain complex 2 (Yield, $80 \%)$.



IR (Nujol): v(B-H) $2500 \mathrm{~cm}^{-1} .{ }^{1} \mathbf{H}$ NMR $\left(500 \mathrm{MHz}, \mathrm{CDCl}_{3}, 25^{\circ} \mathrm{C}\right) \delta: 7.76\left(\mathrm{~s}, 3 \mathrm{H}, \mathrm{H}_{\mathrm{a}}\right)$, $7.20\left(\mathrm{~d}, 6 \mathrm{H}, \mathrm{H}_{\mathrm{d}}\right), 7.10\left(\mathrm{~d}, 6 \mathrm{H}, \mathrm{H}_{\mathrm{c}}\right), 6.10\left(\mathrm{~s}, 3 \mathrm{H}, \mathrm{H}_{\mathrm{b}}\right), 3.02(\mathrm{~m},=\mathrm{CH}), 2.33(\mathrm{~s}, 9 \mathrm{H}, \mathrm{Me})$, $2.24\left(\mathrm{~m}, 1 \mathrm{H},=\mathrm{CH}_{2}\right), 1.32\left(\mathrm{~m}, 1 \mathrm{H},=\mathrm{CH}_{2}\right), 0.73(\mathrm{~s}, 3 \mathrm{H},=\mathrm{CMe}),-1.28\left(\mathrm{~m}, 1 \mathrm{H},=\mathrm{CH}_{2}\right)$, $1.33\left(\mathrm{~m}, 1 \mathrm{H},=\mathrm{CH}_{2}\right) .{ }^{13} \mathbf{C}\left\{{ }^{1} \mathbf{H}\right\} \mathbf{N M R}\left(125 \mathrm{MHz}, \mathrm{CDCl}_{3}, 25^{\circ} \mathrm{C}\right) \delta: 156.2\left(3 \mathrm{C}, \mathrm{C}^{3}\right), 137.2$ $\left(3 \mathrm{C}, \mathrm{C}^{4}\right), 134.7\left(3 \mathrm{C}, \mathrm{CH}^{1}\right), 123.3\left(3 \mathrm{C}, \mathrm{C}^{7}\right), 130.7\left(6 \mathrm{C}, \mathrm{CH}^{6}\right), 127.9\left(6 \mathrm{C}, \mathrm{CH}^{5}\right), 107.7$ $\left(3 \mathrm{C}, \mathrm{CH}^{2}\right), 84.9(=C \mathrm{Me}), 75.0(=\mathrm{CH}), 21.4(3 \mathrm{Me}), 18.2(=\mathrm{CMe}), 10.2\left(=\mathrm{CH}_{2}\right) 1,7.6$ $\left(=\mathrm{CH}_{2}\right)$. Anal. calcd. for $\mathrm{C}_{34} \mathrm{H}_{36} \mathrm{BN}_{6} \mathrm{Ir}$ : C, 56.52; H, 4.88; N, 11.30. Found: C, 57.0; H, $4.9 ; \mathrm{N}, 11.6$.

2.5 Synthesis of complex $\operatorname{Tp}^{\text {tol' }} \operatorname{Ir}(P h)\left(N_{2}\right)(3)$.

Complex $2(0.20 \mathrm{~g}, 0.27 \mathrm{mmol})$ is dissolved in $6 \mathrm{~mL}$ of $\mathrm{C}_{6} \mathrm{H}_{6}$ under a dinitrogen atmosphere and irradiated with UV light for $5 \mathrm{~h}$. The solvent is then removed under vacuum to yield complex $\mathbf{3}$ quantitatively.

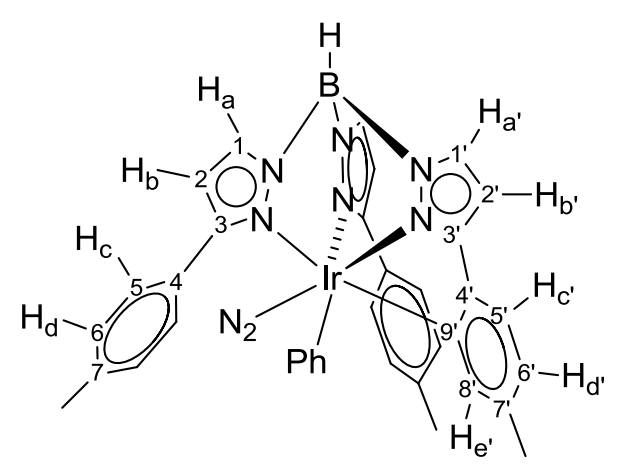

IR (Nujol): $v(\mathrm{~B}-\mathrm{H}) 2480 \mathrm{~cm}^{-1}, v(\mathrm{~N} \equiv \mathrm{N}) 2205 \mathrm{~cm}^{-1} .{ }^{1} \mathbf{H}$ NMR $\left(400 \mathrm{MHz}, \mathrm{C}_{6} \mathrm{D}_{6}, 25{ }^{\circ} \mathrm{C}\right)$

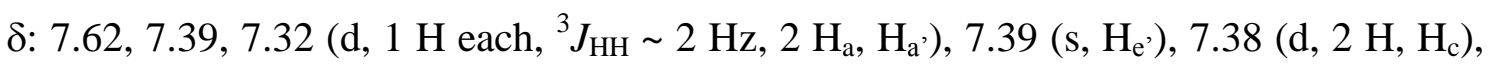
$7.07\left(\mathrm{~d}, 2 \mathrm{H}, \mathrm{H}_{\mathrm{d}}\right), 7.03\left(\mathrm{~d}, 1 \mathrm{H}, \mathrm{H}_{\mathrm{c}^{\prime}}\right), 6.83\left(\mathrm{~d}, 2 \mathrm{H}, \mathrm{H}_{\mathrm{Ph}}\right), 6.71\left(\mathrm{~m}, 6 \mathrm{H}, 3 \mathrm{H}_{\mathrm{Ph}}, 2 \mathrm{H}_{\mathrm{d}}, \mathrm{H}_{\mathrm{d}^{\prime}}\right)$, $6.39\left(\mathrm{~d}, 2 \mathrm{H}, \mathrm{H}_{\mathrm{c}}\right), 6.03,5.95,5.91\left(\mathrm{~d}, 1 \mathrm{H}\right.$ each, ${ }^{3} J_{\mathrm{HH}} \sim 2 \mathrm{~Hz}, 2 \mathrm{H}_{\mathrm{b}}, \mathrm{H}_{\mathrm{b}}$ ) $)$ 2.36, 2.15, 2.00 
(s, $3 \mathrm{H}$ each, Me). ${ }^{13} \mathbf{C}\left\{{ }^{1} \mathbf{H}\right\}$ NMR $\left(100 \mathrm{MHz}, \mathrm{C}_{6} \mathrm{D}_{6}, 25{ }^{\circ} \mathrm{C}\right) \delta: 164.2,157.6,155.9\left(2 \mathrm{C}^{3}\right.$, $\left.\mathrm{C}^{3}\right), 145.8\left(\mathrm{C}^{9^{\prime}}\right), 138.5,137.9\left(2 \mathrm{C}^{7}\right), 137.8,136.7,136.5\left(2 \mathrm{CH}^{1}, \mathrm{CH}^{1}\right), 137.1(3 \mathrm{C}, 2$ $\left.\mathrm{CH}_{\mathrm{Ph}}, \mathrm{C}^{7}\right), 135.4\left(\mathrm{CH}^{8}\right), 130.6\left(2 \mathrm{CH}^{5}\right), 130.4\left(2 \mathrm{CH}^{5}\right), 130.3\left(4 \mathrm{C}, \mathrm{CH}^{5}, \mathrm{C}^{4}\right), 129.0(2$ $\left.\mathrm{CH}^{6}\right), 128.4\left(3 \mathrm{C}, \mathrm{CH}^{6}, \mathrm{C}^{4^{\prime}}\right), 127.5\left(\mathrm{Ir}-\mathrm{C}_{\mathrm{qPh}}\right), 126.9\left(2 \mathrm{CH}_{\mathrm{Ph}}\right), 123.5\left(\mathrm{CH}^{6}\right), 123.0$ $\left(\mathrm{CH}_{\mathrm{Ph}}\right), 122.7\left(\mathrm{CH}^{5}\right), 108.3,105.8,103.1\left(2 \mathrm{CH}^{2}, \mathrm{CH}^{2}\right), 22.2,21.4,21.2(\mathrm{Me})$. Anal. calcd. for $\mathrm{C}_{36} \mathrm{H}_{32} \mathrm{BN}_{8} \mathrm{Ir}$ : C, 55.45; H, 4.14; N, 14.37. Found: C, 55.5; H, 4.3; N, 13.6.

2.6 Synthesis of complex $\operatorname{Tp}^{\text {tol' }} \operatorname{Ir}(\mathrm{Ph})(\mathrm{NCMe})(\mathbf{4})$.

Acetonitrile $(18 \mu \mathrm{L})$ is added to a solution of complex 3 in $6 \mathrm{~mL}$ of $\mathrm{C}_{6} \mathrm{H}_{6}$. The solution is then heated at $60{ }^{\circ} \mathrm{C}$ for 2 and after this time the solvent is removed under vacuum. The solid residue is washed with diethyl ether $(2 \times 5 \mathrm{~mL})$ and dried under vacuum to obtain analytically pure complex 4 (Yield, $60 \%$ ).

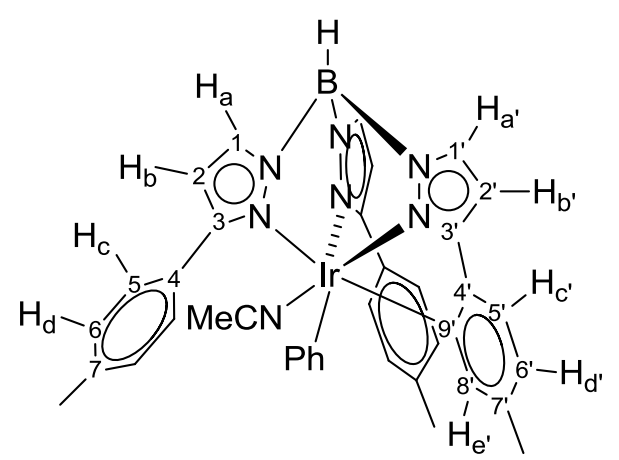

IR (Nujol): $v(\mathrm{~B}-\mathrm{H}) 2470 \mathrm{~cm}^{-1}, v(\mathrm{C} \equiv \mathrm{N}) 2290 \mathrm{~cm}^{-1} .{ }^{\mathbf{1}} \mathbf{H} \mathbf{N M R}\left(500 \mathrm{MHz}, \mathrm{CD}_{2} \mathrm{Cl}_{2}, 25\right.$ $\left.{ }^{\circ} \mathrm{C}\right) \delta: 7.92,7.89,7.61\left(\mathrm{br}, 1 \mathrm{H}\right.$ each, $\left.2 \mathrm{H}_{\mathrm{a}}, \mathrm{H}_{\mathrm{a}}{ }^{\prime}\right), 7.35\left(\mathrm{~d}, 2 \mathrm{H}, \mathrm{H}_{\mathrm{c}}\right), 7.32\left(\mathrm{~s}, 1 \mathrm{H}, \mathrm{H}_{\mathrm{e}}{ }^{\prime}\right), 7.18$ $\left(\mathrm{d}, 1 \mathrm{H}, \mathrm{H}_{\mathrm{c}^{\prime}}\right), 7.14\left(\mathrm{~d}, 2 \mathrm{H}, \mathrm{H}_{\mathrm{d}}\right), 6.74\left(\mathrm{~d}, 2 \mathrm{H}, \mathrm{H}_{\mathrm{d}}\right), 7.74\left(\mathrm{~d}, 1 \mathrm{H}, \mathrm{H}_{\mathrm{d}^{\prime}}\right), 6.67\left(\mathrm{t}, 1 \mathrm{H}, \mathrm{H}_{\mathrm{Ph}}\right)$, 6.60 (m, 4 H, $\mathrm{H}_{\mathrm{Ph}}$ ), 6.23 (d, $2 \mathrm{H}, \mathrm{H}_{\mathrm{c}}$ ), 6.45, 6.14 (br, $3 \mathrm{H}, 2 \mathrm{H}_{\mathrm{b}}, \mathrm{H}_{\mathrm{b}}$ ) , 2.51, 2.39, 2.22 (s, $3 \mathrm{H}$ each, Me), 1.42 (s, $3 \mathrm{H}, \mathrm{NCMe}) .{ }^{13} \mathbf{C}\left\{{ }^{1} \mathbf{H}\right\} \mathbf{N M R}\left(125 \mathrm{MHz}, \mathrm{CD}_{2} \mathrm{Cl}_{2}, 25{ }^{\circ} \mathrm{C}\right) \delta$ : 163.4, 156.2, $154.6\left(2 \mathrm{C}^{3}, \mathrm{C}^{3^{\prime}}\right), 148.3\left(\operatorname{Ir}-\mathrm{C}^{9^{\prime}}\right), 137.8\left(\mathrm{C}^{4^{\prime}}\right), 137.7\left(\mathrm{C}^{7}\right) 137.5,125.7$ (4 $\left.\mathrm{CH}_{\mathrm{Ph}}\right), 137.5,136.3,136.2\left(2 \mathrm{CH}^{1}, \mathrm{CH}^{1}\right), 137.0\left(\mathrm{C}^{7}\right), 136.1\left(\mathrm{C}^{7}\right), 131.1\left(\mathrm{C}^{4}\right), 131.0$ $\left(\mathrm{C}^{4}\right), 130.9\left(\mathrm{Ir}-\mathrm{C}_{\mathrm{Ph}}\right), 130.1\left(2 \mathrm{C}, \mathrm{CH}^{5}\right), 130.0\left(2 \mathrm{C}, \mathrm{CH}^{5}\right), 128.3\left(2 \mathrm{C}, \mathrm{CH}^{6}\right), 127.6(2 \mathrm{C}$, $\left.\mathrm{CH}^{6}\right), 121.8\left(\mathrm{CH}^{6^{\prime}}\right), 121.2\left(2 \mathrm{C}, \mathrm{CH}^{5}, \mathrm{CH}_{\mathrm{Ph}}\right), 114.7(\mathrm{~N} \underline{C M e}), 107.7,105.1,102.5$ (2 $\left.\mathrm{CH}^{2}, \mathrm{CH}^{2}\right), 22.0,21.2,21.0(\mathrm{Me}), 2.45(\mathrm{NC} \underline{\mathrm{Me}})$.

Anal. calcd. for $\mathrm{C}_{38} \mathrm{H}_{35} \mathrm{BN}_{7} \mathrm{Ir}$ : C, 58.53; H, 4.65; N, 10.75. Found: C, 58.6; H, 4.8; N, 10.4 .

2.7 Synthesis of complex $\mathrm{Tp}^{\text {tol' }} \mathrm{Ir}(\mathrm{Ph})\left(\mathrm{CH}_{2}=\mathrm{CH}_{2}\right)(5)$. 
Complex $3(0.2 \mathrm{~g}, 0.26 \mathrm{mmol})$ is dissolved in $\mathrm{C}_{6} \mathrm{H}_{6}(\sim 6 \mathrm{~mL})$ in a thick-wall glass vessel, charged with $2 \mathrm{~atm}$ of ethylene and heated at $60{ }^{\circ} \mathrm{C}$ for $2 \mathrm{~h}$. The solvent is then removed under vacuum to obtain a mixture of $\operatorname{Tp}^{\text {tol' }} \operatorname{Ir}(\mathrm{Ph})\left(\mathrm{CH}_{2}=\mathrm{CH}_{2}\right), \mathbf{5}$, and known $\operatorname{Tp}^{\text {tol" }} \operatorname{Ir}\left(\mathrm{CH}_{2}=\mathrm{CH}_{2}\right), \mathbf{6}$, in a $1: 0.4$ ratio. Complex 5 can be obtained in a pure form by column chromatography, using a mixture of diethyl ether:hexane (10:1) as eluant. Crystals of complex 5 suitable for X-ray studies can be isolated from diethyl ether: hexane mixtures (Yield 40\%).

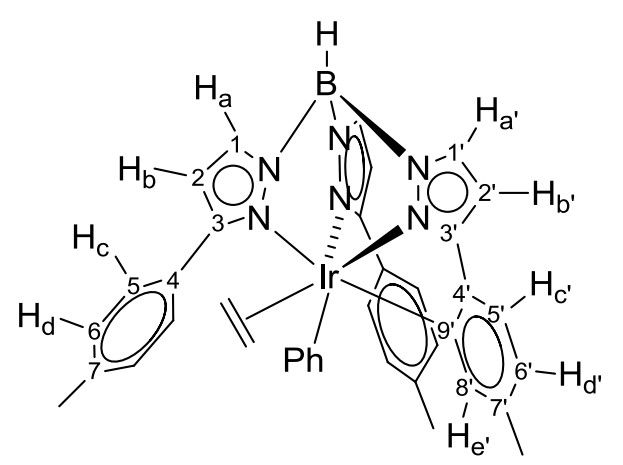

IR (Nujol): $v(\mathrm{~B}-\mathrm{H}) 2525 \mathrm{~cm}^{-1}$. ${ }^{\mathbf{1}} \mathbf{H}$ NMR (400 MHz, $\left.\mathrm{C}_{6} \mathrm{D}_{6}, 25^{\circ} \mathrm{C}\right) \delta: 7.73,7.44,7.35$ $\left(\mathrm{d}, 1 \mathrm{H}\right.$ each, $\left.{ }^{3} J_{\mathrm{HH}} \sim 2 \mathrm{~Hz}, 2 \mathrm{H}_{\mathrm{a}}, \mathrm{H}_{\mathrm{a}}{ }^{\prime}\right), 7.06\left(\mathrm{~d}, 1 \mathrm{H}, \mathrm{H}_{\mathrm{c}^{\prime}}\right), 6.89\left(\mathrm{~d}, 4 \mathrm{H}, \mathrm{H}_{\mathrm{d}}\right), 6.84(\mathrm{~d}, 2 \mathrm{H}, 2$ $\left.\mathrm{H}_{\mathrm{c}}\right), 6.77\left(\mathrm{~d}, 1 \mathrm{H}, \mathrm{H}_{\mathrm{d}^{\prime}}\right), 6.71\left(\mathrm{~m}, 3 \mathrm{H}, \mathrm{H}_{\mathrm{Ph}}\right), 6.57\left(4 \mathrm{H}, 2 \mathrm{H}_{\mathrm{d}}, 2 \mathrm{H}_{\mathrm{c}}\right), 6.54\left(\mathrm{~m}, 2 \mathrm{H}, \mathrm{H}_{\mathrm{Ph}}\right)$, $6.49\left(\mathrm{~s}, 1 \mathrm{H}, \mathrm{H}_{\mathrm{e}}\right.$ ) $)$ 6.02, 5.88, $5.87\left(\mathrm{~d}, 1 \mathrm{H}\right.$ each, ${ }^{3} \mathrm{~J}_{\mathrm{HH}} \sim 2 \mathrm{~Hz}, 2 \mathrm{H}_{\mathrm{b}}, \mathrm{H}_{\mathrm{b}}$ ) $, 3.65,3.27(\mathrm{~m}$, $\mathrm{AA}^{\prime} \mathrm{XX}$ ' system, $\left.=\mathrm{CH}_{2}\right), 2.30,2.13,1.88$ (s, $3 \mathrm{H}$ each, Me) ${ }^{13} \mathbf{C}\left\{{ }^{1} \mathbf{H}\right\} \mathbf{~ N M R}(100 \mathrm{MHz}$, $\left.\mathrm{C}_{6} \mathrm{D}_{6}, 25^{\circ} \mathrm{C}\right) \delta: 162.7,157.4,157.1\left(2 \mathrm{C}^{3}, \mathrm{C}^{3}\right), 142.2\left(\operatorname{Ir}-\mathrm{C}^{9}\right), 138.5\left(\mathrm{C}^{7}\right), 138.4\left(\mathrm{C}^{4}\right)$, $138.5\left(\mathrm{C}^{7}\right), 138.0,136.9,136.2\left(2 \mathrm{CH}^{1}, \mathrm{CH}^{1}\right), 137.6\left(2 \mathrm{CH}_{\mathrm{Ph}}\right), 136.0\left(\mathrm{C}^{7}\right), 132.4$ $\left(\mathrm{CH}^{8^{\prime}}\right), 131.6\left(\mathrm{C}^{4}\right), 131.1\left(\mathrm{C}^{4}\right), 130.7\left(2 \mathrm{CH}^{5}\right), 130.0\left(2 \mathrm{CH}^{5}\right), 128.7\left(2 \mathrm{CH}^{6}\right), 128.4(2$ $\left.\mathrm{CH}^{6}\right), 126.1\left(2 \mathrm{CH}_{\mathrm{Ph}}\right), 123.9\left(\mathrm{Ir}-\mathrm{C}_{\mathrm{qPh}}\right), 123.6\left(\mathrm{CH}^{6}\right), 122.8\left(\mathrm{CH}_{\mathrm{Ph}}\right), 122.5\left(\mathrm{CH}^{5}\right), 109.7$, 107.2, $102.8\left(2 \mathrm{CH}^{2}, \mathrm{CH}^{2}\right), 65.3\left(2 \mathrm{C},=\mathrm{CH}_{2}\right), 22.2,21.4,21.1(\mathrm{Me})$. Anal. calcd. for $\mathrm{C}_{38} \mathrm{H}_{36} \mathrm{BN}_{6} \mathrm{Ir}: \mathrm{C}, 58.53 ; \mathrm{H}, 4.65 ; \mathrm{N}, 10.75$. Found: C, 58.6; H, 4.8; N, 10.4.

\subsection{Synthesis of complex $\operatorname{Tp}^{\text {tol }}$ " $\mathrm{Ir}\left(\mathrm{CH}_{2}=\mathrm{CHC} \equiv \mathrm{CH}\right)(7)$.}

Complex $\operatorname{Tp}^{\text {tol' }} \operatorname{Ir}(\mathrm{Ph})\left(\mathrm{N}_{2}\right)(3)(0.1 \mathrm{~g}, 0.128 \mathrm{mmol})$ is dissolved in $6 \mathrm{~mL}$ of $\mathrm{C}_{6} \mathrm{H}_{6}$ in a thick-wall glass vessel, charged with $2 \mathrm{~atm}$ of $\mathrm{C}_{2} \mathrm{H}_{2}$ and heated at $60{ }^{\circ} \mathrm{C}$ for $1.5 \mathrm{~h}$. The solution is centrifuged, filtered off and evaporated to dryness. The solid residue is dissolved in hexane and crystallized at $-23{ }^{\circ} \mathrm{C}$ (Yield 15\%). 


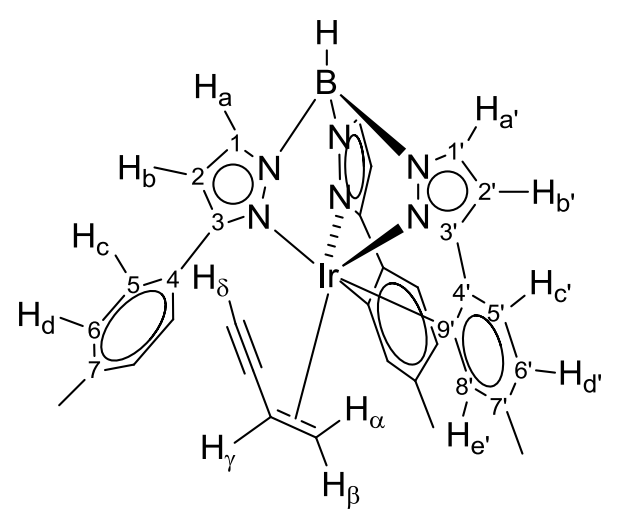

IR (Nujol): $v(\mathrm{~B}-\mathrm{H}) 2480 \mathrm{~cm}^{-1}, v(\mathrm{C} \equiv \mathrm{C}) 2020 \mathrm{~cm}^{-1}$. ${ }^{\mathbf{1}} \mathbf{H}$ NMR $\left(400 \mathrm{MHz}, \mathrm{C}_{6} \mathrm{D}_{6}, 25{ }^{\circ} \mathrm{C}\right)$ $\delta: 7.78,7.48,7.14\left(\mathrm{~d}, 1 \mathrm{H}\right.$ each, $\left.{ }^{3} J_{\mathrm{HH}} \sim 2.4 \mathrm{~Hz}, 2 \mathrm{H}_{\mathrm{a}^{\prime}}, \mathrm{H}_{\mathrm{a}}\right), 7.72\left(\mathrm{br}, \mathrm{H}_{\mathrm{e}}\right), 7.31(\mathrm{~d}, 1 \mathrm{H}$, $\left.\mathrm{H}_{\mathrm{c}^{\prime}}\right), 7.18\left(\mathrm{~d}, 1 \mathrm{H}, \mathrm{H}_{\mathrm{c}^{\prime}}\right), 7.05\left(\mathrm{br} \mathrm{d}, \mathrm{H}_{\mathrm{d}^{\prime}}\right), 6.90\left(\mathrm{~d}, 2 \mathrm{H}, \mathrm{H}_{\mathrm{d}}\right), 6.89\left(\mathrm{br}, 1 \mathrm{H}, \mathrm{H}_{\mathrm{e}^{\prime}}\right), 6.85(\mathrm{~d}, 2$ $\left.\mathrm{H}, \mathrm{H}_{\mathrm{c}}\right), 6.59$ (br d, $\left.\mathrm{H}_{\mathrm{d}}{ }^{\prime}\right), 6.29,5.89,5.86\left(\mathrm{~d}, 1 \mathrm{H}\right.$ each, $\left.{ }^{3} J_{\mathrm{HH}} \sim 2.4 \mathrm{~Hz}, 2 \mathrm{H}_{\mathrm{b}}, \mathrm{H}_{\mathrm{b}}\right), 3.50(\mathrm{~d}$, $\left.1 \mathrm{H},{ }^{3} J_{\mathrm{HH}} \sim 10.5 \mathrm{~Hz}, \mathrm{H}_{\alpha}\right), 3.42\left(\mathrm{~d}, 1 \mathrm{H},{ }^{3} J_{\mathrm{HH}} \sim 9 \mathrm{~Hz}, \mathrm{H}_{\beta}\right), 3.30\left(\mathrm{~m}, \mathrm{H}_{\gamma}\right), 2.55,2.17,1.92$ (s, $3 \mathrm{H}$ each, Me), $1.62\left(\mathrm{~d}, 1 \mathrm{H},{ }^{3} \mathrm{~J}_{\mathrm{HH}} \sim 2 \mathrm{~Hz}, \mathrm{H}_{\delta}\right) .{ }^{13} \mathbf{C}\left\{{ }^{1} \mathbf{H}\right\} \mathbf{N M R}\left(100 \mathrm{MHz}, \mathrm{C}_{6} \mathrm{D}_{6}, 25\right.$ $\left.{ }^{\circ} \mathrm{C}\right) \delta: 164.5,161.8,155.8\left(2 \mathrm{C}^{3}, \mathrm{C}^{3}\right), 145.9\left(\mathrm{Ir}-\mathrm{C}^{9}\right), 141.1,137.9,137.4\left(2 \mathrm{CH}^{1}, \mathrm{CH}^{1}\right)$, 138.5 $\left(\mathrm{CH}^{8^{\prime}}\right), 137.9\left(\mathrm{C}^{7}\right), 137.8\left(\mathrm{C}^{4}\right), 137.5\left(\mathrm{C}^{7^{\prime}}\right), 137.4\left(\mathrm{C}^{4^{\prime}}\right), 137.0\left(\mathrm{C}^{7^{\prime}}\right), 136.4\left(\mathrm{CH}^{8^{\prime}}\right)$, $135.4\left(\mathrm{Ir}-\mathrm{C}^{9}\right), 131.1\left(2 \mathrm{CH}^{5}\right), 130.6\left(\mathrm{C}^{4}\right), 128.7\left(2 \mathrm{CH}^{6}\right), 124.6,124.5\left(2 \mathrm{CH}^{6^{\prime}}\right), 122.9$, $122.7\left(2 \mathrm{CH}^{5}\right), 107.2,104.6,103.3\left(2 \mathrm{CH}^{2}{ }^{\prime}, \mathrm{CH}^{2}{ }^{\prime}\right), 84.2(\underline{C} \equiv \mathrm{CH}), 71.1(\mathrm{C} \equiv \underline{C} H), 60.1$ $\left(=\mathrm{CH}_{2}\right), 54.9(=\mathrm{CH}), 23.2,21.8,21.4(\mathrm{Me})$. HRMS $\mathrm{m} / z$ calcd. for $\mathrm{C}_{34} \mathrm{H}_{30} \mathrm{BN}_{6} \mathrm{Ir}(\mathrm{M}+$ $\mathrm{Na})^{+}$: requires 749.2152, found 749.2155. Crystalline samples of this compound obtained from different preparations give incorrect microanalysis even if they appear to be pure by NMR spectroscopy and provide very accurate mass spectral data. The X-ray analysis herein reported has been performed with this crystalline material.

\section{Results and discussion}

\subsection{Synthesis of $T p^{\text {tol }} R h(I)$ diene complexes}

Encouraged by the remarkable reactivity of rhodium(I) complexes of the auxiliary $\mathrm{Tp}^{\mathrm{Me} 2}$ ligand [3c,7a,c-h,13], we have prepared $\mathrm{Tp}^{\mathrm{tol}} \mathrm{Rh}(\mathrm{I})$ compounds stabilized by coordination of olefins. Reaction of $\mathrm{KTp}^{\text {tol }}$ with $\left[\mathrm{RhCl}\left(\mathrm{C}_{2} \mathrm{H}_{4}\right)_{2}\right]_{2}$, in THF at $0{ }^{\circ} \mathrm{C}$, results in the high yield formation (92\%) of the orange complex $\mathrm{Tp}^{\mathrm{tol}} \mathrm{Rh}\left(\mathrm{C}_{2} \mathrm{H}_{4}\right)_{2}$, 1a (Scheme 1). ${ }^{1} \mathrm{H}$ NMR analysis reveals the existence of two species that are in slow equilibrium in 
solution at room temperature. They form in approximately $1: 1$ ratio. For one of the two isomers the three pyrazolyl rings are equivalent, consistent with $k^{3}-\mathrm{Tp}^{\text {tol }}$ coordination in a highly fluxional five-coordinate structure $[6,14]$. The other isomer shows ${ }^{1} \mathrm{H}$ NMR resonances due to two distinct types of pyrazolyl groups in a $2: 1$ ratio, which indicates a 16-electron, square-planar geometry, with $\kappa^{2}$-coordinated $\mathrm{Tp}^{\text {tol }}$ ligand.



Scheme 1.

As already mentioned the exchange between the two structures is slow, which contrasts with the related complexes $\mathrm{Tp}^{\mathrm{Ms}} \mathrm{Rh}\left(\mathrm{CH}_{2}=\mathrm{C}(\mathrm{R}) \mathrm{C}(\mathrm{Me})=\mathrm{CH}_{2}\right)(\mathrm{R}=\mathrm{H}, \mathrm{Me})$, for which ${ }^{1} \mathrm{H}$ and ${ }^{13} \mathrm{C}\left\{{ }^{1} \mathrm{H}\right\}$ NMR spectra show equivalent pyrazolyl rings at room temperature due to fast exchange in the denticity of the $\mathrm{Tp}^{\mathrm{Ms}}$ ligand. This observation clearly reflects that subtle changes on the substituents of the pyrazolyl groups can favour one of these isomers. Indeed, Moro-oka and co-workers have found that complex $\left[\mathrm{Tp}{ }^{\mathrm{iPr}} \mathrm{Rh}(\mathrm{nbd})\right]$ crystallizes in both $\kappa^{2}$ and $\kappa^{3}$ coordination modes [15].

Two clearly distinguishable IR bands at $c a .2390$ and $2420 \mathrm{~cm}^{-1}$ due to $v(\mathrm{~B}-\mathrm{H})$ are in agreement with the presence of $\kappa^{2}$ and $\kappa^{3}$ complexes [15]. Unfortunately, observation of a very broad ${ }^{11} \mathrm{~B}\left\{{ }^{1} \mathrm{H}\right\}$ NMR signal for complex 1a has prevented us to establish the existence of the two species in solution using as a criterion ${ }^{11} \mathrm{~B}\left\{{ }^{1} \mathrm{H}\right\}$ chemical shift values [16]. However, variable temperature ${ }^{1} \mathrm{H}$ NMR spectra have provided additional information on the $\kappa^{2} \leftrightarrows \kappa^{3}$ equilibrium. Thus, upon cooling from room temperature to $80{ }^{\circ} \mathrm{C}$ the intensity of the signals due to the $\kappa^{2}$ isomer increases at the expense of those of the $\kappa^{3}$ complex, $i$. e., the equilibrium displaces to the side of the $\kappa^{2}$ isomer. At this temperature, the ${ }^{1} \mathrm{H}$ NMR signals for the $\kappa^{3}$ isomer do not split, indicating that fast exchange between its pyrazolyl rings is still occurring. The ratio between species with bidentate and tridentate $\mathrm{Tp}^{\text {tol }}$ depends also on the solvent employed for the NMR 
analysis. Thus, while in deuterated dichloromethane both compounds are present in a ca. $1: 1$ ratio, in $\mathrm{C}_{6} \mathrm{D}_{6}$ the square-planar isomer becomes favoured by a factor of 2 , in agreement with previous work by Venanzi et al. [14].

Complex 1a can be crystallized from diethyl ether solutions at low temperature in the form of single crystals. X-ray diffraction studies reveal $\kappa^{2}$ coordination mode of the $\mathrm{Tp}^{\text {tol }}$ ligand (see Figure 1) and a square planar geometry (sum of angles around $\mathrm{Rh}$ of $358.9^{\circ}$ ). Coordinated pyrazolyl rings have $\mathrm{Rh}-\mathrm{N}$ bond distances of 2.1314(17) and 2.1212(17) $\AA$ while the non-coordinated pyrazolyl group is positioned away from the metal centre. The $\mathrm{Rh} \cdots \mathrm{H}$ distance $(2.70 \AA)$ to the $\mathrm{B}-\mathrm{H}$ terminus is too long to consider any significant electronic interaction [17].

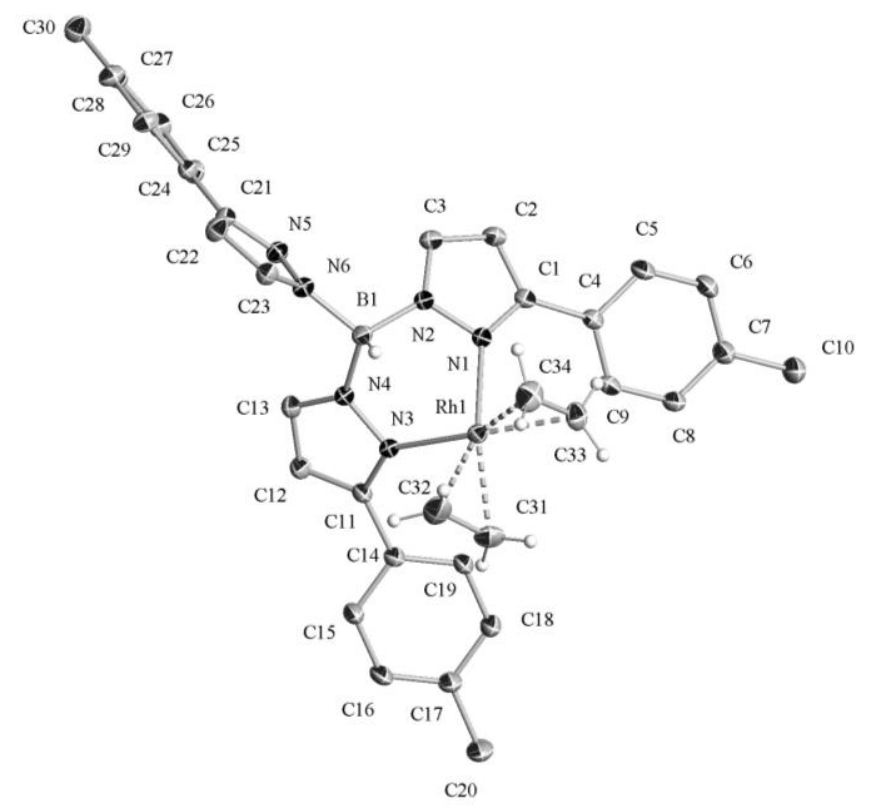

Figure 1. ORTEP view for complex 1a.

Compound $\left[\mathrm{Tp}^{\mathrm{tol}} \mathrm{Rh}\left(\mathrm{CH}_{2}=\mathrm{C}(\mathrm{Me}) \mathrm{C}(\mathrm{Me})=\mathrm{CH}_{2}\right)\right], \mathbf{1 b}$, prepared in the same way as $\mathbf{1 a}$, behaves similarly (Scheme 2). In solution two isomers can be identified in a $2.5: 1$ ratio, once more the $\kappa^{2}$ species prevailing over the $\kappa^{3}$ derivative. In the solid state, the IR spectrum shows two absorptions at $2415 \mathrm{~cm}^{-1}\left(\kappa^{3}\right)$ and $2395 \mathrm{~cm}^{-1}\left(\kappa^{2}\right)$ for the $\mathrm{B}-\mathrm{H}$ stretching vibration, i. e. values identical to those found for $\mathbf{1 a}$. 


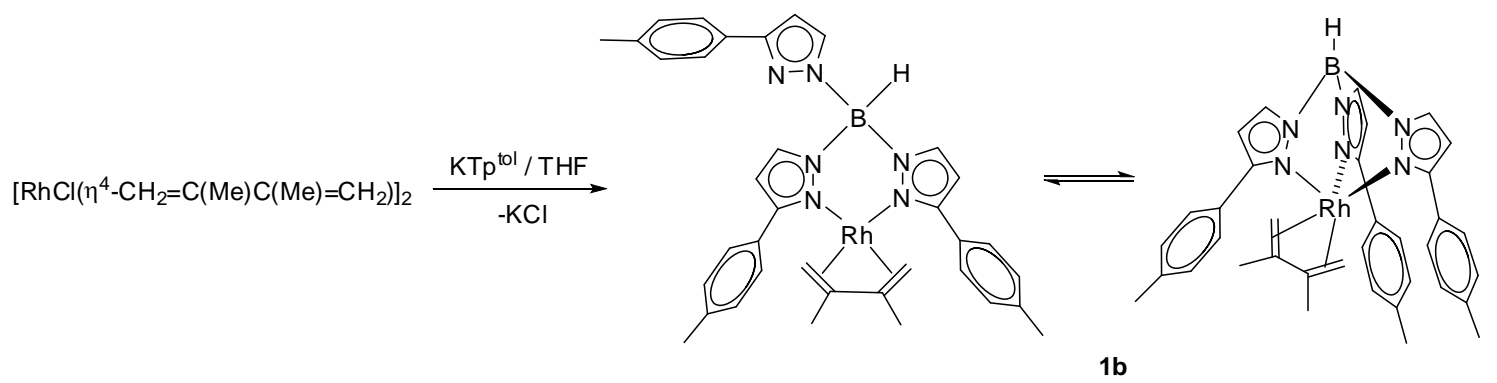

Scheme 2.

Its molecular structure has also been determined by X-ray crystallography (Figure 2). As for $\mathbf{1 a}$, the $\mathrm{T}^{\mathrm{t}}{ }^{\mathrm{ol}}$ ligand in $\mathbf{1} \mathbf{b}$ acts as bidentate, and features metrical parameters very close to those in the bis(ethylene) derivative.

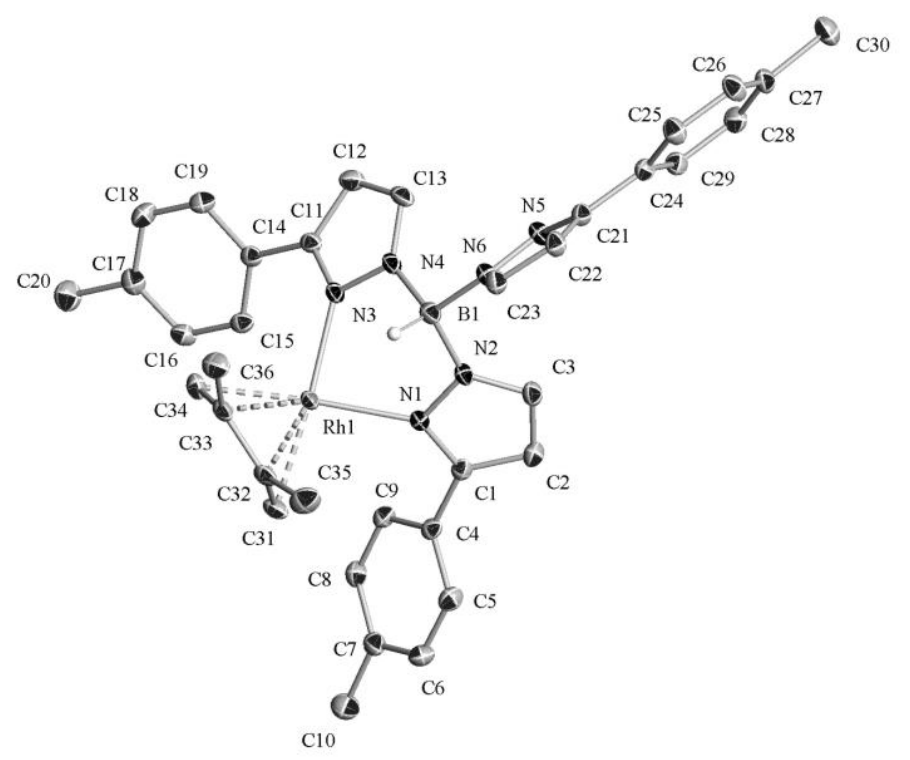

Figure 2. ORTEP view for complex $\mathbf{1 b .}$

\subsection{Synthesis of $T p^{\text {tol }}$ Ir complexes}

Dinitrogen complexes of $\operatorname{TpIr}(\mathrm{III})$ units are useful precursors for various chemical transformations, due in part to facile $\mathrm{N}_{2}$ dissociation, with generation of highly reactive unsaturated fragments. Having in mind the interesting $\mathrm{C}-\mathrm{H}$ bond activation chemistry exhibited by complex $\operatorname{Tp}^{\mathrm{Me} 2} \operatorname{IrPh}_{2}\left(\mathrm{~N}_{2}\right)[9,18]$ and the also attractive, albeit somewhat different chemical behaviour observed for the related $\operatorname{Tp}^{\mathrm{Ms}}{ }^{\prime} \operatorname{Ir}\left(\mathrm{N}_{2}\right)$ [8], we decided to prepare an analogous complex of the metallated $\mathrm{Tp}^{\text {tol }}$ ligand. Following previous 
procedures developed by our group for the synthesis of complexes $\operatorname{Tp}^{\mathrm{Me} 2} \operatorname{IrPh}_{2}\left(\mathrm{~N}_{2}\right)$ and $\mathrm{Tp}^{\mathrm{Ms}}{ }^{\mathrm{N}} \operatorname{Ir}\left(\mathrm{N}_{2}\right), \operatorname{Ir}(\mathrm{I})$ derivative $\mathrm{Tp}^{\mathrm{tol}} \operatorname{Ir}\left(\mathrm{CH}_{2}=\mathrm{C}(\mathrm{Me}) \mathrm{CH}=\mathrm{CH}_{2}\right), 2$, was first formed by reaction of $\mathrm{KTp}^{\text {tol }}$ with in situ generated $\left[\mathrm{IrCl}\left(\mathrm{CH}_{2}=\mathrm{C}(\mathrm{Me}) \mathrm{CH}=\mathrm{CH}_{2}\right)\right]_{2}$ in $80 \%$ yield (Scheme 3). Spectroscopic data are consistent with its formulation as a $\left(\kappa^{3}-\right.$ $\left.\mathrm{Tp}^{\mathrm{tol}}\right) \operatorname{Ir}\left(\mathrm{CH}_{2}=\mathrm{C}(\mathrm{Me}) \mathrm{CH}=\mathrm{CH}_{2}\right)$ compound. In particular, IR spectrum shows an absorption at $2500 \mathrm{~cm}^{-1}$ due to the $\mathrm{B}-\mathrm{H}$ stretching.

When a benzene solution of complex 2 is irradiated with UV light for $5 \mathrm{~h}$, under a dinitrogen atmosphere, a clean reaction occurs that yields the monometallated Ir(III) complex $\left[\left(\kappa^{4}-N, N^{\prime}, N^{\prime}, C-\operatorname{Tp}{ }^{\text {tol' }}\right) \operatorname{Ir}\left(\mathrm{C}_{6} \mathrm{H}_{5}\right)\left(\mathrm{N}_{2}\right)\right], 3$ (Scheme 3$)$. This compound is evidently formed through two $\mathrm{C}-\mathrm{H}$ aryl activation processes. One is intermolecular and involves a molecule of the solvent, $\mathrm{C}_{6} \mathrm{H}_{6}$, while the other is intramolecular and has as key participant one of the $p$-tolyl substituents of the $\mathrm{Tp}^{\text {tol }}$ ligand.
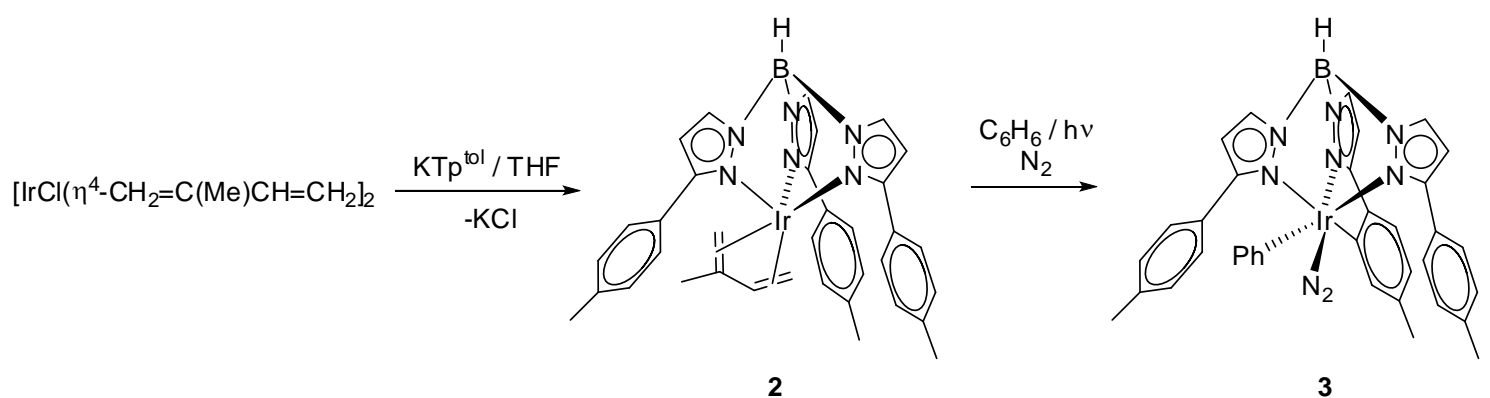

Scheme 3.

Attempts to prepare the doubly metalated $\operatorname{Tp}^{\text {tol" }} \operatorname{Ir}\left(\mathrm{N}_{2}\right)$ derivative have proved unsuccessful. NMR spectroscopic analysis of complex $\mathbf{3}$ is straightforward and reveals its asymmetric nature. Three different types of pyrazolyl groups are clearly discerned in the ${ }^{1} \mathrm{H}$ NMR and ${ }^{13} \mathrm{C}\left\{{ }^{1} \mathrm{H}\right\}$ spectra. The metallated $p$-tolyl group is responsible for a deshielded signal with $\delta 145.8$, attributed to the iridium bound carbon atom, whereas signals due to the $\mathrm{Ir}-\mathrm{C}_{6} \mathrm{H}_{5}$ terminus are clearly discernible in both the ${ }^{1} \mathrm{H}$ and the ${ }^{13} \mathrm{C}\left\{{ }^{1} \mathrm{H}\right\}$ NMR spectra. The IR spectrum shows characteristic bands assigned to the $v(\mathrm{~B}-\mathrm{H})$ and $v(\mathrm{~N}-\mathrm{N})$ stretching vibrations at $2480 \mathrm{~cm}^{-1}$ and $2205 \mathrm{~cm}^{-1}$, respectively. The first is suggestive of a $\mathrm{k}^{3}-N, N^{\prime}, N^{\prime}$ ' coordination of the $\mathrm{Tp}^{\text {tol }}$ ligand, which as already indicated has undergone monometallation and is therefore coordinating to iridium in a tetradentate fashion. In turn, the band at $2205 \mathrm{~cm}^{-1}$ demonstrates that the sixth coordination site of the $\operatorname{Ir}($ III) complex is occupied by a molecule of dinitrogen. This band appears at somewhat higher wavenumber than in the analogous complex 
$\operatorname{Tp}^{\mathrm{Me} 2} \operatorname{Ir}\left(\mathrm{C}_{6} \mathrm{H}_{5}\right)_{2}\left(\mathrm{~N}_{2}\right), \quad\left(2190 \mathrm{~cm}^{-1}\right.$, [18a] $)$ and $\mathrm{Tp}^{\mathrm{Ms}}{ }^{\prime \prime} \operatorname{Ir}\left(\mathrm{N}_{2}\right), \quad\left(2180 \mathrm{~cm}^{-1}, \quad\right.$ [51]; $\mathrm{Tp}^{\mathrm{Ms} "}$ represents the doubly metallated $\mathrm{Tp}^{\mathrm{Ms}}$ ligand, and $\mathrm{Tp}^{\mathrm{Ms}}$ is hydrotris(mesitylpyrazolyl)borate).

Characterization of compound $\mathbf{3}$ by X-ray crystallography was of interest, as there are few dinitrogen complexes of $\operatorname{Ir}(\mathrm{III})$ that have been structurally authenticated by X-ray diffraction [19]. For $\operatorname{Tp}^{\mathrm{Me} 2} \operatorname{Ir}(\mathrm{III})$ only the binuclear $\left[\mathrm{Tp}^{\mathrm{Me} 2} \operatorname{Ir}\left(\mathrm{C}_{6} \mathrm{H}_{5}\right)_{2}\right]_{2}\left(\mu-\mathrm{N}_{2}\right)$ species provided crystals suitable for X-ray studies [18a], whereas in the case of $\operatorname{Tp}^{\mathrm{Ms}} \operatorname{Ir}\left(\mathrm{N}_{2}\right)$, that contains a doubly metallated $\mathrm{Tp}^{\mathrm{Ms}}$ ligand, no single crystals could be isolated. Structural information on the latter compound was obtained indirectly from knowledge of the structure of the NCMe adduct, $\mathrm{Tp}^{\mathrm{Ms}}$ Ir(NCMe), that results from its reaction with acetonitrile. Failure to obtain binuclear complexes within the $\mathrm{Tp}^{\text {tol' }} \mathrm{Ir}(\mathrm{III})$ and $\mathrm{Tp}^{\mathrm{Ms}}$ 'Ir(III) systems stems probably from steric hindrance between aryl substituents of the hydrotris(pyrazolyl)borate ligands.

As can be seen in Figure 3, compound $\mathbf{3}$ is mononuclear and features a linear Ir-N-N functionality $\left(\mathrm{Ir}-\mathrm{N}-\mathrm{N}=177^{\circ}\right.$ ), which is additionally characterized by an $\mathrm{Ir}-\mathrm{N}$ bond distance of 1.946(4) $\AA$, practically identical to the Ir-N separations of 1.93(2) $\AA$ found in binuclear $\left[\mathrm{Tp}^{\mathrm{Me} 2} \operatorname{Ir}\left(\mathrm{C}_{6} \mathrm{H}_{5}\right)_{2}\right]_{2}\left(\mu-\mathrm{N}_{2}\right)$ [18a]. The $\mathrm{N}-\mathrm{N}$ bond length of 1.089(5) $\AA$ in $\mathbf{3}$, is comparable to the $1.097 \AA$ value characteristic of free $\mathrm{N}_{2}$. Interestingly, the two Ir-N bonds to pyrazolyl groups trans to the two aryl fragments, with lengths of 2.174(3) $\AA$ (average) are longer by $0.2 \AA$ than Ir-N bond trans to dinitrogen. This is a clear reflection of the poor donor properties of the $\mathrm{N}_{2}$ ligand and consequently of its low trans influence. 


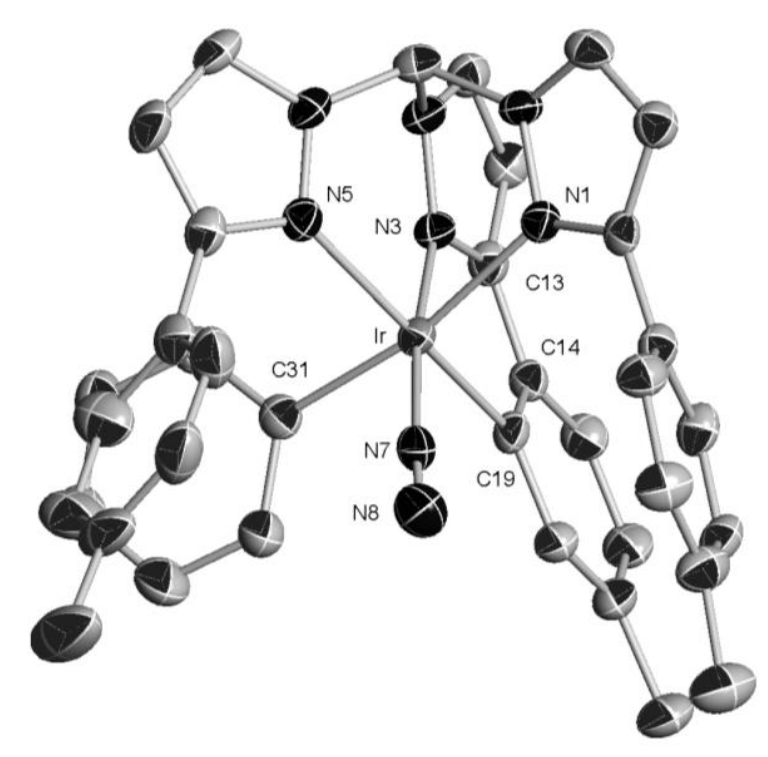

Figure 3. ORTEP view for complex 3.

Not unexpectedly, the $\mathrm{N}_{2}$ ligand in complex $\operatorname{Tp}^{\text {tol' }} \operatorname{Ir}\left(\mathrm{C}_{6} \mathrm{H}_{5}\right)\left(\mathrm{N}_{2}\right), 3$, is rather labile, and requires only moderate heating for its dissociation to yield unsaturated $\operatorname{Ir}(\mathrm{III})$, 16electron species, that can be trapped by action of Lewis bases. For example, when complex 3 is heated at $60{ }^{\circ} \mathrm{C}$ for $2 \mathrm{~h}$ in benzene in the presence of acetonitrile, a clean reaction occurs with formation of the expected acetonitrile adduct 4 (Scheme 4). The identity of $\mathbf{4}$ has been ascertained by spectroscopic and analytical data. Its IR spectrum shows a medium intensity band at $2290 \mathrm{~cm}^{-1}$ for the $\mathrm{C} \equiv \mathrm{N}$ stretching. In the ${ }^{1} \mathrm{H} N M R$ spectrum a signal at $1.42 \mathrm{ppm}$ has been assigned to the coordinated molecule of acetonitrile. Two ${ }^{13} \mathrm{C}\left\{{ }^{1} \mathrm{H}\right\}$ NMR resonances with chemical shift values of 114.7 and 2.45 , indicate further the presence of acetonitrile. Comparative kinetic studies on the



4

Scheme 4. 
reactions of compound 3 and $\operatorname{Tp}^{\mathrm{Me}} \operatorname{Ir}\left(\mathrm{C}_{6} \mathrm{H}_{5}\right)_{2}\left(\mathrm{~N}_{2}\right)$ with acetonitrile reveal that $\mathrm{N}_{2}$ displacement in the two complexes occurs with comparable rates.

Ethylene is also able to substitute the $\mathrm{N}_{2}$ ligand in $\mathbf{3}$ with formation of the corresponding adduct, 5 (Scheme 5). However, in this case the known ethylene adduct $\operatorname{Tp}^{\text {tol" }} \operatorname{Ir}\left(\mathrm{C}_{2} \mathrm{H}_{4}\right)$, 6 [6], that contains a $\mathrm{Tp}^{\text {tol }}$ ligand metallated in two $p$-tolyl substituents forms also, with an approximate $5: 6$ ratio of $1: 0.4$. Prolonged heating of this mixture ( $c a .24 \mathrm{~h}$ ) does not change the ratio of the products. Moreover, the two compounds can be separated taking advantage of their different solubilities in diethyl ether and when pure $\mathbf{5}$ is heated under the above conditions $\left(60{ }^{\circ} \mathrm{C}, 2 \mathrm{~h}\right)$ conversion to 6 is not detected. Nevertheless, under more forcing conditions $\left(80-100{ }^{\circ} \mathrm{C}, 24 \mathrm{~h}\right)$ the second metallation of the $\mathrm{Tp}^{\text {tol }}$ ligand occurs and $\mathbf{6}$ becomes the only reaction product.

The different outcomes of the reactions of 3 with $\mathrm{NCMe}$ and $\mathrm{C}_{2} \mathrm{H}_{4}$ can be ascribed to the capacity of the Lewis base to trap the five-coordinate intermediate $\left[\operatorname{Tp}^{\text {tol' }} \operatorname{Ir}\left(\mathrm{C}_{6} \mathrm{H}_{5}\right)\right]$ that results from $\mathrm{N}_{2}$ dissociation from complex 3 . NCMe reacts very quickly with this intermediate and forms adduct $\mathbf{4}$ as the only reaction product. In contrast with this, in the ethylene case $\left[\mathrm{Tp}^{\text {tol' }} \operatorname{Ir}\left(\mathrm{C}_{6} \mathrm{H}_{5}\right)\right]$ has a sufficiently long life to undergo intramolecular $\mathrm{C}-\mathrm{H}$ activation of an ortho $\mathrm{C}-\mathrm{H}$ bond in one of the two tolyl rings that are still free. A molecule of $\mathrm{C}_{6} \mathrm{H}_{6}$ is extruded and the resulting [Tp ${ }^{\text {tol" }} \mathrm{Ir}$ ] species reacts then with $\mathrm{C}_{2} \mathrm{H}_{4}$ to form 6 .

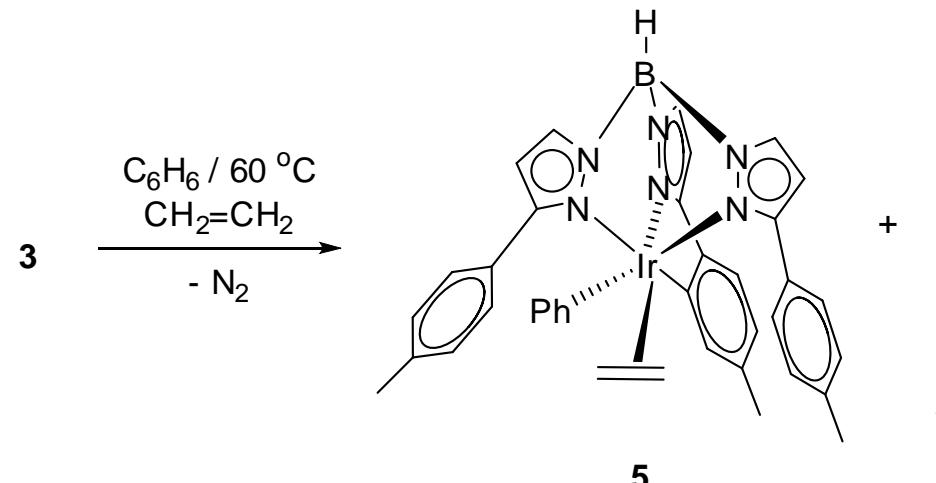

5



6

Scheme 5.

X-ray diffraction analysis of $\mathbf{6}$ has already been performed [6]. Concentrated solutions of 5 in diethyl ether cooled at $-10{ }^{\circ} \mathrm{C}$ provide adequate crystals for crystallographic studies. Figure 4 shows an ORTEP view of a molecule of this complex. It features an $\mathrm{Ir}-\mathrm{C}$ bond length to the metallated ring of 2.038(2) $\AA$, which is somewhat shorter than 
the $\mathrm{Ir}-\mathrm{C}_{6} \mathrm{H}_{5}$ bong length of 2.079(2) $\AA$, and compares well with $\mathrm{Ir}-\mathrm{C}$ separations found in other structurally characterized iridium complexes of metallated $\mathrm{Tp}^{\text {tol }}$ ligands [6].

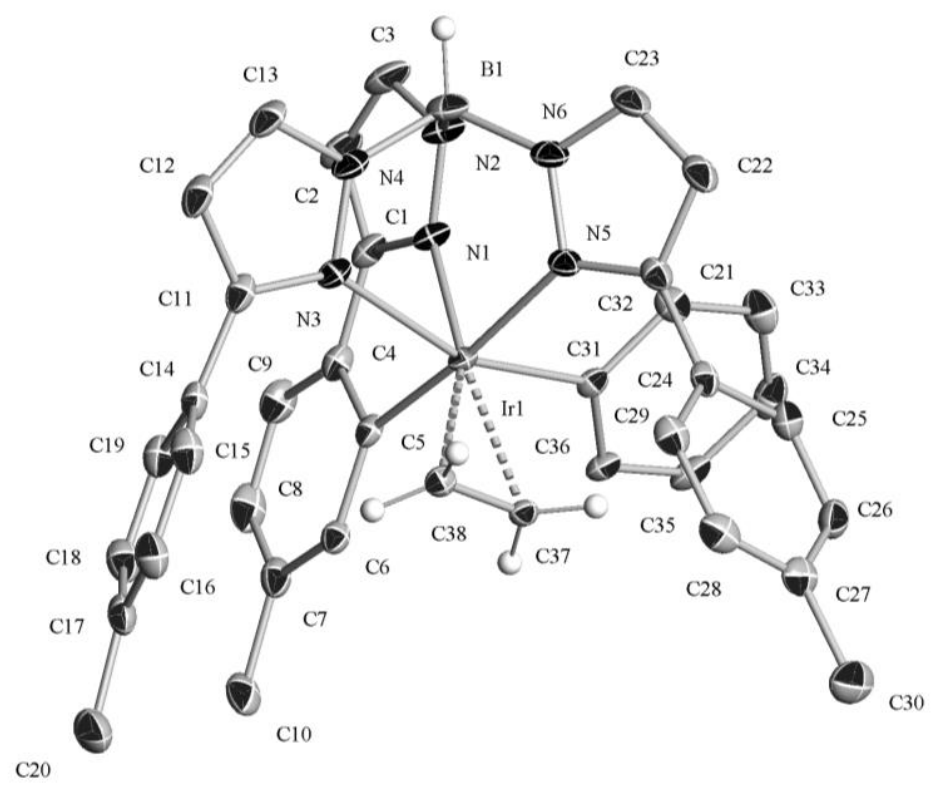

Figure 4. ORTEP view for complex 5.

We have recently shown that addition of acetylene to dinitrogen complex $\operatorname{Tp}{ }^{M s "} \operatorname{Ir}\left(\mathrm{N}_{2}\right)$ (where $\mathrm{Tp}^{\mathrm{Ms}}$ " represents the doubly metallated $\mathrm{Tp}^{\mathrm{Ms}}$ ligand) at $60{ }^{\circ} \mathrm{C}$ leads to a vinylacetylene complex $\operatorname{Tp}^{\mathrm{Ms}}{ }^{\prime \prime} \operatorname{Ir}\left(\mathrm{H}_{2} \mathrm{C}=\mathrm{CH}-\mathrm{C} \equiv \mathrm{CH}\right)$, in which the enyne is coordinated to iridium through the carbon-carbon double bond [8b]. To assess whether dimerization of acetylene by $\operatorname{Tp} \operatorname{Ir}(\mathrm{III})$ complexes is a general process and if coordination of the generated vinylacetylene through the double bond is preferred thermodynamically, complex 3 has been heated $\left(60{ }^{\circ} \mathrm{C}\right)$ under an acetylene atmosphere (2 atm). A new complex 7 containing a coordinated molecule of vinylacetylene forms as a single product, with no indication being inferred regarding formation of a $\pi$-acetylene compound similar to complex 6 (Scheme 6). ${ }^{1} \mathrm{H}$ and ${ }^{13} \mathrm{C}\left\{{ }^{1} \mathrm{H}\right\}$ NMR data hint at coordination of the unsaturated hydrocarbon through the olefinic moiety. The $=\mathrm{CH}_{2}$ protons of the alkene resonate as doublets at $3.50\left(\mathrm{H}_{\alpha}\right)$ and $3.42\left(\mathrm{H}_{\beta}\right)$ 

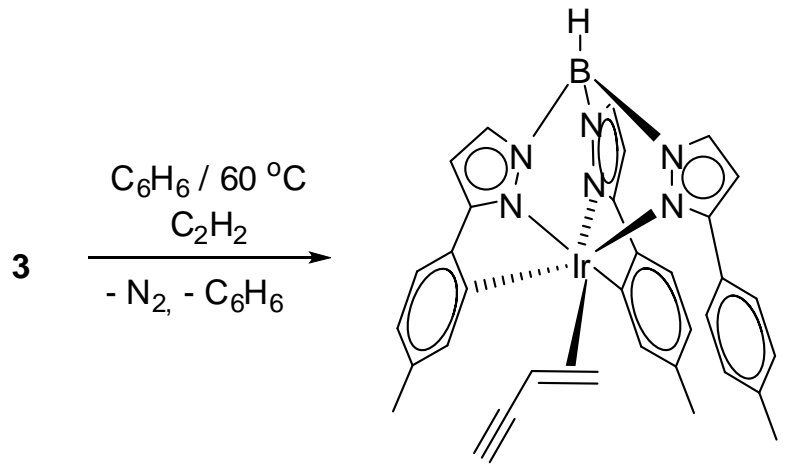

7

Scheme 6.

ppm whereas the $=\mathrm{CH}$ resonance appears as a multiplet at $3.30\left(\mathrm{H}_{\gamma}\right)$ ppm. These chemical shift values are up-field $(\sim 2 \mathrm{ppm})$ in comparison with those of free vinylacetylene [20], as expected for olefinic protons bonded to transition metals. The $\mathrm{C} \equiv \mathrm{C}-\mathrm{H}$ proton also appears shifted up-field with respect to free terminal alkynes $(\sim 1$ ppm). The ${ }^{13} \mathrm{C}\left\{{ }^{1} \mathrm{H}\right\}$ NMR spectrum supports also coordination of the double bond to the iridium centre. Two signals at $\delta 84.2$ and 71.1 are recorded for the internal and terminal carbon nuclei of the triple bond, respectively, while the olefinic carbon atoms resonate at $60.1\left(=\mathrm{CH}_{2}\right)$ and $54.9(=\mathrm{CH})$ ppm. Another interesting piece of information that can be drawn from the NMR analysis is that the $\mathrm{Tp}^{\text {tol }}$ ligand has undergone metallation of a second tolyl fragment during reaction, and therefore behaves as $\kappa^{5}$.

The structure of complex 7 has been unequivocally established by X-ray diffraction studies (Figure 5). The Ir-C separations to the double bond have values of 2.147(2) and 2.169(2) $\AA$ for the terminal (C31) and internal (C32) carbon atoms, respectively. The C31-C32, C32-C33 and C33-C34 bond distances are 1.396(3), 1.435(3) and 1.186(4) $\AA$. All these values are identical, within experimental error, to those found in the analogous complex $\operatorname{Tp}^{\mathrm{Ms}}{ }^{\prime} \operatorname{Ir}\left(\mathrm{H}_{2} \mathrm{C}=\mathrm{CHC} \equiv \mathrm{CH}\right)[8 \mathrm{~b}]$. 


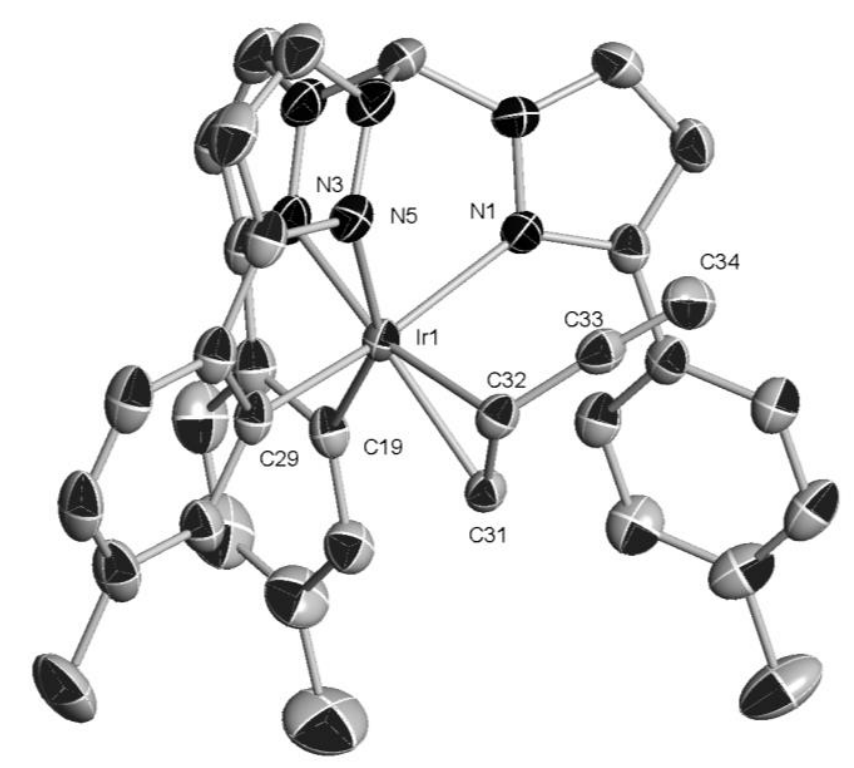

Figure 5. ORTEP view for complex 7.

Formation of complex 7 implies $\mathrm{C}-\mathrm{H}$ activation and $\mathrm{C}-\mathrm{C}$ coupling reactions between two molecules of acetylene but, as in the case of $\operatorname{Tp}^{\mathrm{Ms}}{ }^{\prime \prime} \operatorname{Ir}\left(\mathrm{H}_{2} \mathrm{C}=\mathrm{CHC} \equiv \mathrm{CH}\right)$, no intermediates where detected in the course of the reaction. In view of the complexity of the latter system, no mechanistic studies on the reaction of $\mathbf{3}$ with $\mathrm{C}_{2} \mathrm{H}_{2}$ have been executed.

\section{Conclusions}

Complexes $\mathrm{Tp}^{\text {tol }} \mathrm{Rh}$ (olefin) $)_{2}$ are readily accessible by the straightforward reaction of $\mathrm{KTp}^{\text {tol }}$ with the corresponding $\left[\mathrm{RhCl}(\text { olefin })_{2}\right]_{2}$ starting material. Spectroscopic data for these complexes indicate that an equilibrium between $\kappa^{2}$ and $\kappa^{3}$ coordination modes is occurring in solution. The analogous iridium complex $\operatorname{Tp}^{\mathrm{tol}} \operatorname{Ir}\left(\mathrm{CH}_{2}=\mathrm{C}(\mathrm{Me}) \mathrm{CH}=\mathrm{CH}_{2}\right)$ has also been prepared in a similar way and shown to be an excellent precursor for the synthesis of the $\operatorname{Ir}(\mathrm{III})$ dinitrogen complex $\operatorname{Tp}^{\text {tol' }} \operatorname{Ir}\left(\mathrm{C}_{6} \mathrm{H}_{5}\right)\left(\mathrm{N}_{2}\right)$, in which the $\mathrm{Tp}^{\text {tol }}$ is monometallated, acting therefore as a $\kappa^{4}$ ligand. This compound is one of the few $\operatorname{Ir}(\mathrm{III})$ dinitrogen complexes that have been isolated and crystallographicaly characterized. Its reactivity towards acetonitrile, ethylene and acetylene has been explored and has revealed that $\mathrm{N}_{2}$ dissociation to yield transient, unsaturated 16-electron species $\left[\mathrm{Tp}^{\text {tol' }} \operatorname{Ir}\left(\mathrm{C}_{6} \mathrm{H}_{5}\right)\right]$ is an easy process. It has also been observed that metallation of one of the still non-metallated tolyl groups can take place during these reactions to give access to a $\kappa^{5}$ coordination mode of the $\mathrm{Tp}^{\text {tol }}$ ligand. 


\section{Acknowledgements}

Financial support from the Spanish Ministerio de Educación y Ciencia (MEC) (Project No. CTQ2007-62814) and Consolider-Ingenio 2010 (No. CSD2007-00006) (FEDER support), the Junta de Andalucía (Project Nos. FQM-3151 and FQM-672) is gratefully acknowledge. A.C.E. and J.E.V.V. thanks the CONACYT (Ref. No. 229340) and the MEC (F.P.U) for research grants.

\section{Appendix A. Supplementary material.}

CCDC 791539, 791540, 791573, 791541 and 791574 contain the supplementary crystallographic data for complexes $\mathbf{1 a}, \mathbf{1 b}, \mathbf{3}, \mathbf{5}$ and $\mathbf{7}$. These data can be obtained free of charge from the Cambridge Crystallographic Data Centre via www.ccdc.cam.ac.uk/data_request/cif.

\section{References}

[1] (a) S. Trofimenko, Scorpionates-The Coordination Chemistry of Polypyrazolylborate Ligand; Imperial College Press: London, 1999. (b) C. Pettinari, Scorpionates II: Chelating Borate Ligands; Imperial College Press: London, 2008.

[2] S. Trofimenko, J. Am. Chem. Soc. 88 (1966) 1842.

[3] (a) C. Slugovc, I. Padilla-Martínez, S. Siriol, E. Carmona, Coord. Chem. Rev. 213 (2001) 129;

(b) F.T. Edelmann, Angew.Chem. Int. Ed. 40 (2001) 1656;

(c) I.R. Crossley, Adv. Organomet. Chem. 56 (2008) 199.

[4] (a) M. Paneque, S. Siriol, M. Trujillo, E. Gutiérrez-Puebla, M.A. Monge, E. Carmona, Angew. Chem. Int. Ed. 39 (2000) 218;

(b) M. Paneque, S. Siriol, M. Trujillo, E. Carmona, E. Gutiérrez-Puebla, A. Monge, C. Ruiz, F. Malbosc, C. Serra-Le Berre, P. Kalck, M. Etienne, J.C. Daran, Chem. Eur. J. 7 (2001) 3869.

[5] (a) N. Kitajima, M. Osawa, M. Tanaka, Y. Moro-oka, J. Am. Chem. Soc. 113 (1991) 8952;

(b) A.J. Amoroso, J.C. Jeffery, P.L. Jones, J.A. McCleverty, E. Psillakis, M.D. Ward, J. Chem. Soc.; Chem. Commun. (1994), 2751; 
(c) A.J. Amoroso, J.C. Jeffery, P.L. Jones, J.A. McCleverty, L. Rees, A.L. Rheingold, Y. Sun, J. Takats, S. Trofimenko, M.D. Ward, G.P.A. Yap, J. Chem. Soc.; Chem. Commun. (1995) 1881;

(d) M.C. Keyes, V.G. Young, W.B. Tolman, Organometallics 15 (1996) 4133;

(e) J.L. Jones, K.L.V. Mann, C. Jeffery, J.A. McCleverty, M.D. Ward, Polyhedron 16 (1997) 2435;

(f) Y. Takahashi, S. Hikichi, M. Akita, Y. Moro-oka, Chem. Commun. (1999) 1491.

(g) Y. Takahashi, S. Hikichi, A. Akita, Y. Moro-oka, Organometallics 18 (1999) 2571;

(h) A.L. Rheingold, B.S. Haggerty, L.M. Liable-Sands, S. Trofimenko, Inorg. Chem. 38 (1999) 6306;

(i) S. Slugovc, K. Mereiter, S. Trofimenko, E. Carmona, Chem. Commun. (2000), 121 ;

(j) C. Slugovc, K. Mereiter, S. Trofimenko, E. Carmona, Helv. Chim. Acta 84 (2001) 2868;

(k) Y. Takahashi, M. Hashimoto, S. Hikichi, M. Akita, Y. Moro-oka, Inorg. Chim. Acta 357 (2004) 1711.

(1) J.A. López, K. Mereiter, M. Paneque, M.L. Poveda, O. Serrano, S. Trofimenko,

E. Carmona, Chem. Commun. (2006) 3921.

[6] S. Conejero, A.C. Esqueda, J.E.V. Valpuesta, Inorg. Chim. Acta 362 (2009) 4539.

[7] (a) M.C. Asplund, P.T. Snee, J.S. Yeston, M.J. Wilkens, C.K. Payne, H. Yang, K.T. Kotz, H. Frei, R.G. Bergman, C.B. Harris, J. Am. Chem. Soc. 124 (2002) 10605;

(b) E. Carmona, M. Paneque, L.L. Santos, V. Salazar, Coord. Chem. Rev. 249 (2005) 1729;

(c) A.J. Vetter, R.D. Rieth, W.D. Jones, Proc. Natl. Acad. Sci. 104 (2007) 6957;

(d) A.J. Blake, M.W. George, M.B. Hall, J. McMaster, P. Portius, X.Z. Sun, M. Towrie, C.E. Webster, C. Wilson, S.D. Zarić, Organometallics 27 (2008) 189;

(e) M.E. Evans, C.L. Burke, S. Yaibuathes, E. Clot, O, Eisenstein, W.D. Jones, J. Am. Chem. Soc. 131 (2009) 13464;

(f) D.D. Wick, W.D. Jones, Inorg. Chim. Acta 362 (2009) 4416;

(g) M.E. Evans, T. Li, A.J. Vetter, R.D Rieth, W.D. Jones, J. Org. Chem. 74 (2009) 6907 ;

(h) A.J. Vetter, R.D. Rieth, W.W. Brennessel, W.D. Jones, J. Am. Chem. Soc. 131 (2009) 10742; 
(i) S. Conejero, L.L. Santos, M. Paneque, M.L. Poveda, E. Carmona, Acc. Chem. Res. 43 (2010) 572.

(j) C. Slugovc, K. Mereiter, S. Trofimenko, E. Carmona, Angew. Chem. Int. Ed. 39 (2000) 2158.

[8] (a) E. Alvarez, S. Conejero, P. Lara, J.A. López, M. Paneque, A. Petronilho, M.L. Poveda, D. del Rio, O. Serrano, E. Carmona, J. Am. Chem. Soc. 129 (2007) 14130;

(b) P. Lara, J. López-Serrano, C. Maya, M. Paneque, M.L. Poveda, L.J. Sánchez, J.E.V. Valpuesta, E. Carmona, Organometallics 28 (2009) 4649;

[9] (a) E. Alvarez, S. Conejero, M. Paneque, A. Petronilho, M.L. Poveda, O. Serrano, E. Carmona, J. Am. Chem. Soc. 128 (2006) 13060;

(b) S. Conejero, P. Lara, M. Paneque, A. Petronilho, M.L. Poveda, O. Serrano, F. Vattier, E. Alvarez, C. Maya, V. Salazar, E. Carmona, Angew. Chem. Int. Ed. 47 (2008) 4380.

[10] R. Cramer, Inorg. Synth. 15 (1974) 14.

[11] J.L. Herde, J.C. Lambert, C.V. Senoff, Inorg.Chem. 15 (1974) 19.

[12] S. Trofimenko, J.C. Calabrese, J.K. Kochi, S. Wolowiec, F.B. Hulsbergen, J. Reedijk, Inorg. Chem. 31 (1992) 3943;

[13] (a) P.J. Pérez, M.L. Poveda, E. Carmona, Angew. Chem. Int. Ed. 34 (1995) 231;

(b) M. Paneque, S. Taboada, E. Carmona, Organometallics 15 (1996) 2678;

(c) M. Paneque, P.J. Pérez, A. Pizzano, M.L. Poveda, S. Taboada, M. Trujillo, E. Carmona, Organometallics 18 (1999) 4304;

(d) M. Nicasio, M. Paneque, P.J. Pérez, A. Pizzano, M.L. Poveda, L. Rey, S. Sirol, S. Taboada, M. Trujillo, A. Monge, C. Ruiz, E. Carmona, Inorg. Chem. 39 (2000) 180.

[14] U.E. Bucher, A. Currao, R. Nesper, M. Rueggen, L.M. Venanzi, E. Younger, Inorg. Chem. 34 (1995) 66.

[15] M. Akita, K. Ohta, Y. Takahashi, S. Hikichi, Y. Moro-oka, Organometallics 16 (1997) 4121.

[16] T.O. Northcutt, R.J. Lachicotte, W.D. Jones, Organometallics 17 (1998) 5148.

[17] G.R. Desiraju, Dalton Trans. (2000) 3745.

[18] (a) E. Gutiérrez, A. Monge, M.C. Nicasio, M.L. Poveda, E. Carmona, J. Am. Chem. Soc. 116 (1994) 791;

(b) M. Paneque, M.L. Poveda, L.L. Santos, E. Camona, A. Lledós, G. Ujaque, K. Mereiter, Angew. Chem. Int. Ed. 43 (2004) 3708; 
(c) M. Paneque, M.L. Poveda, L.L. Santos, V. Salazar, E. Carmona, Chem. Commun. (2004) 1838;

(d) P. Lara, M. Paneque, M.L. Poveda, V. Salazar, L.L. Santos, E. Carmona, J. Am. Chem. Soc. 128 (2006) 3512;

(e) P. Lara, M. Paneque, M.L. Poveda, L.L. Santos, J.E.V. Valpuesta, V. Salazar, E. Carmona, S. Moncho, G. Ujaque, A. Lledós, C. Maya, K. Mereiter, Chem. Eur. J. 15 (2009) 9046;

(f) P. Lara, M. Paneque, M.L. Poveda, L.L. Santos, J.E.V. Valpuesta, V. Salazar, E. Carmona, S. Moncho, G. Ujaque, A. Lledós, E. Alvarez, K. Mereiter, Chem. Eur. J. 15 (2009) 9034.

[19] D.M. Tellers, R.G. Bergman, J. Am. Chem. Soc. 122 (2000) 954.

[20] T.-S. Peng, Y. Wang, A.M. Arif, J.A. Gladysz, Organometallics 12 (1993) 4535. 